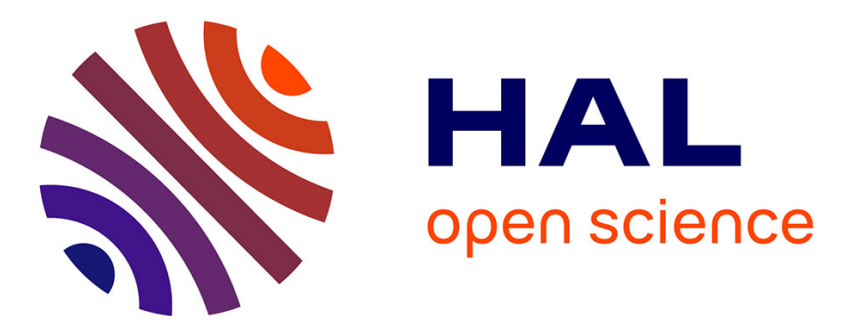

\title{
High-order numerical scheme for compressible multi-component real gas flows using an extension of the Roe approximate Riemann solver and specific Monotonicity-Preserving constraints
}

Luc Lecointre, Ronan Vicquelin, Sergey Kudriakov, Etienne Studer, Christian

Tenaud

\section{To cite this version:}

Luc Lecointre, Ronan Vicquelin, Sergey Kudriakov, Etienne Studer, Christian Tenaud. High-order numerical scheme for compressible multi-component real gas flows using an extension of the Roe approximate Riemann solver and specific Monotonicity-Preserving constraints. Journal of Computational Physics, In press, 10.1016/j.jcp.2021.110821 . hal-03451235

\author{
HAL Id: hal-03451235 \\ https://hal.science/hal-03451235
}

Submitted on 26 Nov 2021

HAL is a multi-disciplinary open access archive for the deposit and dissemination of scientific research documents, whether they are published or not. The documents may come from teaching and research institutions in France or abroad, or from public or private research centers.
L'archive ouverte pluridisciplinaire HAL, est destinée au dépôt et à la diffusion de documents scientifiques de niveau recherche, publiés ou non, émanant des établissements d'enseignement et de recherche français ou étrangers, des laboratoires publics ou privés. 


\title{
High-order numerical scheme for compressible multi-component real gas flows using an extension of the Roe approximate Riemann solver and specific Monotonicity-Preserving constraints.
}

\author{
Luc Lecointre*a,b,c , Ronan Vicquelin ${ }^{b}$, Sergey Kudriakova ${ }^{\mathrm{a}}$, Etienne Studer ${ }^{\mathrm{a}}$, \\ Christian Tenaud ${ }^{\mathrm{b}}$ \\ ${ }^{a}$ Université Paris-Saclay, CEA, Service de Thermo-hydraulique et de Mécanique des \\ Fluides, 91191, Gif-sur-Yvette, France. \\ ${ }^{b}$ Université Paris-Saclay, CNRS, CentraleSupélec, Laboratoire EM2C, 91190, \\ Gif-sur-Yvette, France. \\ ${ }^{c}$ Université Paris-Saclay, CNRS, Laboratoire Interdisciplinaire des Sciences du Numérique, \\ 91400, Orsay, France.
}

\begin{abstract}
The purpose of this paper is to develop a high-order shock-capturing scheme capable of predicting flows where shock waves with high-temperature jumps interact with multi-component real gas mixtures, assuming a local thermodynamic equilibrium. We first propose a generalization of the Roe solver for distinct species with non-ideal thermodynamic properties that relies on the original method proposed by Vinokur \& Montagné [1]. This method uses an approximation of compressibility factors to estimate a coherent value of the speed of sound at the Roe averaged state.
\end{abstract}

This Roe averaged state is introduced in the One-Step Monotonicity-Preserving (OSMP) scheme, originally developed by Daru and Tenaud [2], to obtain an extension to the high-order with Lax-Wendroff procedure adequate for dealing with non-ideal gas flows. To avoid thermodynamic inconsistencies in the evolution of the Roe average state over a large stencil, we propose to reformulate the discrete total energy flux of the initial solver. This new formulation uses a combination of Riemann invariants related to the species mass fractions and avoids the influence of the independent values of the compressibility factors in the total energy flux computation. An additional M-P constraint on this new combination allows dealing with discontinuities. Based on the averaged speed of sound estimated by our proposed extension of the Vinokur \& Montagné method, we demonstrate that this new formulation is equivalent to selecting a new combination of compressibility factors that completely fulfil the jump relationships of the Riemann problem.

To properly capture discontinuities while optimizing the number of numerical

*corresponding author. E-mail address: luc.lecointre@centraliens.net 
cells, the new high-order OSMP scheme is combined with an Adaptive Multiresolution 3 procedure to automatically refine grid in regions where steep gradients occur and coarsen grid elsewhere. The order of the numerical method is evaluated on the convection of density and mass fraction waves. Its capability of capturing discontinuities is validated on a 1-D shock tube problem with a mixture of Nitrogen, Oxygen and dense refrigerant R22 gases. We show that smooth solutions, as well as discontinuities, are recovered with high accuracy. The 2-D interaction between a shock wave in Air with a cylindrical bubble initially filled with dense refrigerant R22 gas is also considered. Present results compare very well with both a recent fully resolved numerical solution of ideal gases and experimental results obtained with real gases. Compared to ideal gas solutions corresponding to calorically perfect gas, drastic changes are recorded on the predicted temperature and the bubble flow patterns that fully justify the use of relevant thermodynamics and the proposed numerical method to account for real gas properties.

Keywords: High-order numerical scheme, Monotonicity-Preserving constraints, Multi-component Real Gas Flow, Roe approximate Riemann solver

\section{Introduction}

Studying compressible multi-component real gas flows is of engineering interest since they arise in numerous applications. They occur in the aerodynamic domain with supersonic and hypersonic flows [4, and in many combustion applications, [5] especially in the safety area dealing with reacting flows with potential explosions. Simulation of such compressible multi-component flows is a challenging task since they generally involve shock waves interacting with contact discontinuities or component interfaces that produce acoustic waves as well as vortical structures through baroclinic effects. Unsteady flow phenomena then occur at various time and length scales.

For predicting fast dynamic small-scale structures with accuracy, it is mandatory to use high-order schemes on refined grids in regions where vortical phenomena and discontinuities occur. However, high-order schemes are known to produce spurious oscillations in the vicinity of stiff gradients (known as the Gibb's phenomenon). Shock-capturing procedures must be used to avoid such spurious oscillations.

Riemann approximate solvers are known to solve hyperbolic problems where discontinuities occur. The Roe solver [6] is often privileged since it is the less diffusive among all Riemann solvers because it is based on the complete characteristic waves of the exact Riemann problem. Originally, the Roe solver was built for calorically perfect gas [6], based on an assumption of relatively small temperature variations. However, in hypersonic flow or detonation cases, significant temperature variations in mixtures with several species must be considered, and an ideal gas assumption cannot be regarded. In fact, the vibrational degrees of freedom of polyatomic molecules must be taken into account to get accurate results. This is generally done by considering the dependency of the specific heat capacities of the species with respect to the temperature. Therefore, extending the Roe solver to multi-component mixtures with specific and distinct 
physical and thermodynamic properties for each species is mandatory.

Introducing the assumption of local equilibrium, which considers that thermodynamics of the gas mixture depends on two independent variables, for instance, the density and the internal energy, several generalizations of the Roe approximate Riemann solver have been proposed in cases with mono-component real gases [7, 8, 9, 10, 11, 12. Difficulties usually encountered in developing such generalizations come from the non-uniqueness of the Roe averaged state at the interface between right and left states of the Riemann problem when applied to a non-ideal gas or a non-perfect equation of state. Indeed, the Roe averaged state requires additional approximations for the compressibility factors, which are pressure derivatives with respect to the independent variables (the density and the internal energy, for instance). Several methods to approximate those pressure derivatives have already been formulated in the literature. A solution proposed in [8] consists in considering arithmetic averages of the pressure derivatives of fictitious intermediary states corresponding to the different possible paths between the left and right states. In [13], the solution is obtained by neglecting some Riemann invariants. However, such methods generally give poor results or even be out of the range of the equation of state. Since the end of the 90s, a large part of the generalization of the Roe solver to real gases is based on a projection of approximated pressure derivatives onto a subspace defined by the jump relationships across the interface [9, 10, 11. A Roe averaged speed of sound is then deduced from the Roe average of pressure derivatives. An evaluation of such methods has been performed by Mottura et al. [14, who conclude that similar results with the different methods are obtained with, however, a better numerical efficiency with the methods proposed by Vinokur \& Montagné [9] and Liou, Van Leer and Shuen [10]. More recently, contrary to the usual strategy, Arabi et al. [12] proposed to first approximate the speed of sound before computing interface averages of the corresponding compressibility factors. Later on, Arabi et al. 15] extended the proposed Roe scheme for multi-component real gas flows by treating the mixture as a single equilibrium gas to define the contact discontinuity and thus the density jump. Knowing that the mass fractions are constant on both sides of the contact discontinuity, the jump of the convective partial masses can be calculated using the classical resolution of a Riemann problem. The advantage of this formulation is to guarantee the positivity of the mass fractions, but the mass flux computation is at this point only first-order accurate. Extension to a higher-order numerical scheme with quadratic reconstruction or MUSCL scheme [16] are evoked. However, the successive Roe average states can then become inconsistent with the thermodynamic properties evolution along a large stencil, and the sum of the mass fractions can differ from unity when using more than two species. Besides, a solution to compute a real gas mixture with two distinct species was also proposed in [17] with an extension to high-order approximations. Nevertheless, this is only applicable to mixtures with at most two distinct species.

These previous studies are a strong motivation to develop a high-order accurate numerical scheme based on a Roe scheme for multi-component real gas flows. This paper aims to investigate the additional constraints needed to develop a stable high-order Roe scheme capable of predicting flows where strong shock waves with large temperature jumps interact with mixtures of several species. 
We here follow techniques introduced by Vinokur and Montagné 9 to evaluate a Roe averaged value of compressibility factors in a non-thermally perfect single gas. In this paper, we first propose extending these techniques to multicomponent real gas flows based on a similar prediction from an integral between the left and the right states of the Riemann problem, followed by an orthogonal projection onto a well-chosen subspace. It allows us to define an accurate Roe averaged value of the speed of sound. Continuing the work done by Daru \& Tenaud [2, 18, in the development of high-order accurate schemes based on a Lax-Wendroff approach for unsteady shocked thermally perfect gas flow predictions, we secondly introduce the proposed averaged state of the compressibility factors into the One-Step Monotonicity-Preserving (OSMP) scheme to obtain an extension towards real gas flows. However, numerical artifacts appear in the vicinity of contact discontinuity (interface between species), and potential low order of convergence is observed in smooth solutions with large variations of composition and temperature. Those issues are found to come from the proposed compressibility factor averaged states of the Riemann problem that do not seem consistent over the large stencil when using a high-order scheme. By analyzing Riemann invariants, we propose a reformulation of the discrete total energy flux using a linear combination of Riemann invariants associated with the same characteristic wave to recover high-order accuracy. An additional Monotonicity-Preserving constraint is applied to this linear combination to deal with discontinuities. Knowing the averaged speed of sound evaluated by our proposed extension of the Vinokur and Montagné method [9], this new formulation gives a supplementary relation between compressibility factors. It enforces the jump relationships to be satisfied across the interface between the left and right states of the Riemann problem. The proposed approach is then validated on well-documented 1-D and 2-D test cases.

To get a sharp resolution of discontinuities and stiff gradient while saving grid points in smooth regions, this high-order numerical scheme is combined with an Adaptive Multiresolution procedure to automatically concentrate grid points in regions where steep gradients occur and coarsen mesh elsewhere.

The paper is organized as follows. Governing equations are presented in section $\S 2$ The formalism used for applying the Roe solver to real gases is described in $\S 3$ and an extension of the Roe scheme to multi-component real gases is proposed. In section $\S 4$, we present the high-order numerical scheme with flux limiter based on Monotonicity-Preserving constraints. This OSMP scheme is applied to a particular combination of the Riemann invariants involved in the discrete total energy flux. Section $\S 5$ is devoted to the validation of the proposed approach. A convergence study is performed on the convection of mass fraction and density waves to illustrate the efficiency of the new formulation. 1-D shock tube problems with and without real gas effect are then applied. Comparisons with the exact solution of the Riemann problem validate the present approach to deal with discontinuities. Then, the 2-D interaction of a shock wave in Air with a cylindrical bubble initially filled with dense refrigerant R22 gas is considered. Present results are compared to a recent numerical solution of ideal gases as well as results with real gas experiments. We finally performed simulations of a real gas configuration with larger temperature variations that provide original results we expect could constitute reference results. We conclude and draw up some perspectives to this work $(\S 6)$. 


\section{Governing equations}

We consider a multi-component fluid with $n_{s}$ different species. Under the conservative form, the Euler equation is written as:

$$
\frac{\partial \mathbf{U}}{\partial t}+\nabla \cdot \mathbf{F}(\mathbf{U})
$$

In the following, we will denote $N$ the total number of equations solved; i.e. $N=n_{s}+2$ in 1-D, and $N=n_{s}+4$ in 3-D.

$\mathbf{U}$ is the vector of the $N$ conservative variables, and $\mathbf{F}(\mathbf{U})$ is the Euler flux vector:

$$
\mathbf{U}=\left[\begin{array}{c}
\rho_{1} \\
\vdots \\
\rho_{n_{s}} \\
\rho \mathbf{v} \\
\rho E
\end{array}\right], \quad \mathbf{F}(\mathbf{U})=\left[\begin{array}{c}
\rho_{1} \mathbf{v} \\
\vdots \\
\rho_{n_{s}} \mathbf{v} \\
\rho \mathbf{v} \otimes \mathbf{v}+p \mathbb{I} \\
\rho \mathbf{v} H
\end{array}\right],
$$

$\rho$ is the density, $\rho_{i}=\rho Y_{i}$ where $Y_{i}$ is the mass fraction of the $i^{t h}$ species, $\mathbf{v}$ is the velocity vector, $p$ is the static pressure of the mixture, $E$ the total energy per unit of mass, and $H=E+p / \rho$ is the total specific enthalpy.

We denote the specific internal energy $e=E-E_{c}$, where $E_{c}$ is the kinetic energy, i.e. $E_{c}=\frac{1}{2} \mathbf{v} \cdot \mathbf{v}$. In this study, we assume the local equilibrium state and the pressure is related to the partial density and the internal energy density, noted $\epsilon=\rho e$. A general form of the equation of state is:

$$
p=p\left(\rho_{1}, \rho_{2}, \ldots, \rho_{n_{s}}, \epsilon\right) .
$$

Though a general form is employed, we assume the convexity of the equation of state to guarantee the existence and unicity of the weak solution of the Euler hyperbolic system of conservation laws.

The differential of $p$ is written by using the compressibility coefficients $\kappa$ and $\chi_{i}, i=1, \ldots, n_{s}$ :

$$
\mathrm{d} p=\sum_{i} \chi_{i} \mathrm{~d} \rho_{i}+\kappa \mathrm{d} \epsilon,
$$

that are expressed as:

$$
\chi_{i}=\left.\frac{\partial p}{\partial \rho_{i}}\right|_{\rho_{k, k \neq i}, \epsilon} \quad \text { and } \quad \kappa=\left.\frac{\partial p}{\partial \epsilon}\right|_{\rho_{i, i=1, \ldots, n_{s}}} .
$$

If $h=e+p / \rho$ is the specific enthalpy, the sound speed $c_{s}$ can then be obtained by:

$$
c_{s}^{2}=\sum_{i} \chi_{i} Y_{i}+\kappa h,
$$

\section{Extension of the Roe scheme for multi-component real gases}




\subsection{Riemann approximated solver of Roe}

The objective is to extend the Roe solver for multi-component real gases.

First, we would like to introduce the classic Roe solver to explain how the multicomponent nature of the gas comes into play. To ease reading, the Roe solver is presented on a $1 \mathrm{D}$ Euler equation. The quasi-linear form of (1) is then as follows:

$$
\frac{\partial \mathbf{U}}{\partial t}+\mathbf{A}(\mathbf{U}) \cdot \frac{\partial \mathbf{U}}{\partial x}=0
$$

A stands for the Jacobian matrix of the Euler flux.

Following an approximate Riemann problem, the principle of the Roe solver is to find an intermediate state $\widetilde{\mathbf{U}}$ of the vector of the conservative variables between the left $\left(\mathbf{U}_{L}\right)$ and the right $\left(\mathbf{U}_{R}\right)$ states. Then, the Jacobian matrix at the intermediate state can be expressed as:

$$
\mathbf{A}(\tilde{\mathbf{U}})=\widetilde{\mathbf{A}}\left(\mathbf{U}_{L}, \mathbf{U}_{R}\right) .
$$

Based on the expression of the compressibility factors (5), the Jacobian matrix $\widetilde{\mathbf{A}}$ of the Euler flux at the Roe averaged state is:

$$
\mathbf{A}(\tilde{\mathbf{U}})=\left(\begin{array}{ccccccc}
\tilde{u}\left(1-\tilde{Y}_{1}\right) & -\tilde{u} \tilde{Y}_{1} & \ldots & \ldots & -\tilde{u} \tilde{Y}_{1} & \tilde{Y}_{1} & 0 \\
-\tilde{u} \tilde{Y}_{2} & \tilde{u}\left(1-\tilde{Y}_{2}\right) & -\tilde{u} \tilde{Y}_{2} & \cdots & -\tilde{u} \tilde{Y}_{2} & \tilde{Y}_{2} & 0 \\
\vdots & \ddots & \ddots & \ddots & \vdots & \vdots & \vdots \\
\vdots & & \ddots & \ddots & -\tilde{u} \tilde{Y}_{n_{s}-1} & \tilde{Y}_{n_{s}-1} & 0 \\
-\tilde{u} \tilde{Y}_{n_{s}} & \ldots & \cdots & -\tilde{u} \tilde{Y}_{n_{s}} & \tilde{u}\left(1-\tilde{Y}_{n_{s}}\right) & \tilde{Y}_{n_{s}} & 0 \\
\tilde{\chi} 1+\tilde{\kappa} \tilde{E}_{c}-\tilde{u}^{2} & \tilde{\chi}_{2}+\tilde{\kappa} \tilde{E}_{c}-\tilde{u}^{2} & \cdots & \cdots & \tilde{\chi} n_{s}+\tilde{\kappa} \tilde{E}_{c}-\tilde{u}^{2} & (2-\tilde{\kappa}) \tilde{u} & \tilde{\kappa} \\
\tilde{u}\left(\tilde{\chi}_{1}+\tilde{\kappa} \tilde{E}_{c}-\tilde{H}\right) & \tilde{u}\left(\tilde{\chi}_{2}+\tilde{\kappa} \tilde{E}_{c}-\tilde{H}\right) & \cdots & \cdots & \tilde{u}\left(\tilde{\chi} n_{s}+\tilde{\kappa} \tilde{E}_{c}-\tilde{H}\right) \tilde{H}-\tilde{\kappa} \tilde{u}^{2} & \tilde{u}(1+\tilde{\kappa})
\end{array}\right) .
$$

According to [6], in order to apply the Roe solver, several properties must be fulfilled by the Jacobian matrix $\widetilde{\mathbf{A}}$ :

(i) $\widetilde{\mathbf{A}}\left(\mathbf{U}_{L}, \mathbf{U}_{R}\right)$ is a mapping from the vector space $\mathbf{U}$ to the vector space $\mathbf{F}(\mathbf{U})$

(ii) The system must be hyperbolic, i.e. the eigenvalues $\widetilde{\lambda}_{k}(k \in[1, N])$ are real and the eigenvectors $\widetilde{\mathbf{K}}^{(k)}$ are linearly independent;

(iii) The approximation is consistent with the Jacobian matrix, i.e. as $\mathbf{U}_{L}, \mathbf{U}_{R}$ smoothly tends to $\mathbf{U}, \widetilde{\mathbf{A}}\left(\mathbf{U}_{L}, \mathbf{U}_{R}\right)$ smoothly tends to the Jacobian $\mathbf{A}(\mathbf{U})$;

(iv) The conservation must be satisfied across discontinuities, i.e. $\widetilde{\mathbf{A}}\left(\mathbf{U}_{L}, \mathbf{U}_{R}\right) \cdot\left(\mathbf{U}_{L}-\mathbf{U}_{R}\right)=\mathbf{F}\left(\mathbf{U}_{L}\right)-\mathbf{F}\left(\mathbf{U}_{R}\right)$.

With such a Jacobian matrix, the numerical flux $\widetilde{\mathbf{F}}\left(\mathbf{U}_{L}, \mathbf{U}_{R}\right)$ at the interface between two consecutive cells then becomes:

$$
\widetilde{\mathbf{F}}\left(\mathbf{U}_{L}, \mathbf{U}_{R}\right)=\frac{1}{2}\left(\mathbf{F}\left(\mathbf{U}_{L}\right)+\mathbf{F}\left(\mathbf{U}_{R}\right)\right)-\frac{1}{2} \sum_{k=1}^{N} \widetilde{\alpha}_{k} \cdot\left|\widetilde{\lambda}_{k}\right| \widetilde{\mathbf{K}}^{(k)},
$$


with $\widetilde{\lambda}_{k}=\widetilde{\lambda}_{k}\left(\mathbf{U}_{L}, \mathbf{U}_{R}\right)$ and $\widetilde{\mathbf{K}}^{(k)}=\widetilde{\mathbf{K}}^{(k)}\left(\mathbf{U}_{L}, \mathbf{U}_{R}\right)$ are respectively the $k^{\text {th }}$ eigenvalues and the corresponding $k^{\text {th }}$ eigenvectors of the matrix. Eigenvalues $\widetilde{\lambda_{k}}$ are arranged in a diagonal matrix $\boldsymbol{\Lambda}$ :

$$
\boldsymbol{\Lambda}=\left(\begin{array}{cccccc}
\widetilde{u}, & & & & & \\
& \widetilde{u} & & & & \\
& & \ddots & & & \\
& & \widetilde{u} & & \\
& & & \widetilde{u}+\widetilde{c}_{s} & \\
& & & & & \widetilde{u}-\widetilde{c}_{s}
\end{array}\right) .
$$

The corresponding eigenvectors are given by:

$$
\begin{aligned}
& \mathbf{K}^{(1)}=\left[1,0, \ldots, \ldots, 0, \widetilde{u}, \widetilde{E}_{c}-\widetilde{\chi}_{1} / \widetilde{\kappa}\right]^{T} ; \\
& \mathbf{K}^{(i)}=\left[0, \ldots, 1, \ldots, 0, \widetilde{u}, \widetilde{E}_{c}-\widetilde{\chi}_{i} / \widetilde{\kappa}\right]^{T} ; \\
& \mathbf{K}^{\left(n_{s}\right)}=\left[0, \ldots, \ldots, 0,1, \widetilde{u}, \widetilde{E}_{c}-\widetilde{\chi}_{n_{s}} / \widetilde{\kappa}\right]^{T} ; \\
& \mathbf{K}^{(N-1)}=\left[\widetilde{Y}_{1}, \ldots, \widetilde{Y}_{i}, \ldots, \widetilde{Y}_{n_{s}}, \widetilde{u}+\widetilde{c}_{s}, \widetilde{H}+\widetilde{u} \widetilde{c}_{s}\right]^{T} ; \\
& \mathbf{K}^{(N)}=\left[\widetilde{Y}_{1}, \ldots, \widetilde{Y}_{i}, \ldots, \widetilde{Y}_{n_{s}}, \widetilde{u}-\widetilde{c}_{s}, \widetilde{H}-\widetilde{u} \widetilde{c}_{s}\right]^{T} .
\end{aligned}
$$

The $\widetilde{\alpha}_{k}$ is the Riemann invariant corresponding to the $k^{t h}$ component of $\left(\mathbf{U}_{R}-\right.$ $\mathbf{U}_{L}$ ) in the eigenvector basis.

$\mathbf{U}_{R}-\mathbf{U}_{L}=\sum_{k=1}^{N} \widetilde{\alpha}_{k} \cdot \widetilde{\mathbf{K}}^{(k)} \quad$ or equivalently $\quad\left(\begin{array}{c}\widetilde{\alpha}_{1} \\ \vdots \\ \alpha_{N}\end{array}\right)=\widetilde{\mathbf{K}}^{-1} \cdot\left(\mathbf{U}_{R}-\mathbf{U}_{L}\right)$,

with $\widetilde{\mathbf{K}}^{-1}$ the inverse matrix of $\widetilde{\mathbf{K}}$

\subsection{Roe averaged operator}

At this step, no assumption is made on the equation of state except on the general form described by (3). However, if the equation of state is such that pressure derivatives (5) exist, then the first two properties of the linearized Jacobian matrix $\widetilde{\mathbf{A}}\left(\mathbf{U}_{L}, \mathbf{U}_{R}\right)$ at the Roe averaged state are satisfied. For the last two properties, Roe [6] suggests using a parameter vector $\mathbf{Q}$ such that components of $\mathbf{U}$ and of the Euler flux vector $\mathbf{F}(\mathbf{U})$ can be expressed as quadratic compositions of the $\mathbf{Q}$ components. In the case of multi-component flow, this corresponds to:

$$
\mathbf{Q}=\left(q_{1}, q_{2}, \ldots, q_{N}\right)^{T}=\sqrt{\rho}\left(Y_{1}, \ldots, Y_{n_{s}}, u, H\right)^{T} .
$$

The averaged vector $\widetilde{\mathbf{Q}}$ is obtained with a simple arithmetic averaging $\widetilde{\mathbf{Q}}=$ $\frac{1}{2}\left(\mathbf{Q}_{R}+\mathbf{Q}_{L}\right)$. The corresponding variable of mass fraction $\widetilde{Y}_{1}, \ldots, \widetilde{Y}_{n_{s}}$, velocity $\widetilde{u}$ and enthalpy $\widetilde{H}$ at the Roe averaged state are then computed with the Roe 
averaged operator, where $r$ stands for each component of $\left(Y_{1}, \ldots, Y_{n_{s}}, u, H\right)^{T}$ :

$$
\widetilde{r}=\theta r_{L}+(1-\theta) r_{R}
$$

with

$$
\theta=\frac{\sqrt{\rho_{L}}}{\sqrt{\rho_{L}}+\sqrt{\rho_{R}}}
$$

and the value of the density at the interface becomes:

$$
\widetilde{\rho}=\sqrt{\rho_{L} \rho_{R}} .
$$

Let us consider $\phi$ and $\psi$ two arbitrary variables. The Roe averaged operator respects the following identities for the variation across interface $\Delta(\cdot)=(\cdot)_{R}-$ $(\cdot)_{L}:$

$$
\begin{aligned}
\Delta(\rho \phi) & =\widetilde{\rho} \Delta \phi+\widetilde{\phi} \Delta \rho ; \\
\Delta(\rho \psi \phi) & =\widetilde{\rho} \widetilde{\phi} \Delta \psi+\widetilde{\rho} \widetilde{\psi} \Delta \phi+\widetilde{\phi} \widetilde{\psi} \Delta \rho .
\end{aligned}
$$

\subsection{Expression of the compressibility factors at the Roe averaged state}

In the multi-component case with non-ideal gases, to completely define the Jacobian matrix (9), we need to determine intermediate values of the compressibility factors at the Roe averaged state. Combining the property (iv) of the Roe solver and identities (18), a new relationship between the variation of conservative variables and the compressibility factors is established through the use of the Roe averaged operator:

$$
\Delta p=\sum_{i=1}^{n_{s}} \widetilde{\chi}_{i} \Delta \rho_{i}+\widetilde{\kappa} \Delta \epsilon
$$

We here extend to the case of multi-component real gas flow the procedure introduced by Montagné et al. [1] and modified by Liou et al. [10. It consists in obtaining at first predicted values of the compressibility factors by using the left and right thermodynamic states, then correct secondly these predictions by projection onto the $\left(n_{s}-1\right)$ hyperplane defined by the relationship (19).

Following Vinokur and Montagné, the prediction $\left(\widehat{\chi}_{i}, \widehat{\kappa}\right)$ of the compressibility factors is fulfilled by integrating themselves along a straight-line path between the two left and right thermodynamic states:

$$
\begin{aligned}
\widehat{\chi}_{i} & =\int_{0}^{1} \chi_{i}\left[\epsilon(\zeta), \rho_{1}(\zeta), \ldots, \rho_{n_{s}}(\zeta)\right] d \zeta, \\
\widehat{\kappa} & =\int_{0}^{1} \kappa\left[\epsilon(\zeta), \rho_{1}(\zeta), \ldots, \rho_{n_{s}}(\zeta)\right] d \zeta .
\end{aligned}
$$

where the parameter $\zeta$ is normalized such that $\zeta_{L}=0$ and $\zeta_{R}=1$ and the straight line path is defined with:

$$
\begin{aligned}
\rho_{i}(\zeta) & =\rho_{i, L}+\zeta \Delta \rho_{i}, \quad i=1, \ldots, n_{s} \\
\epsilon(\zeta) & =\epsilon_{L}+\zeta \Delta \epsilon
\end{aligned}
$$




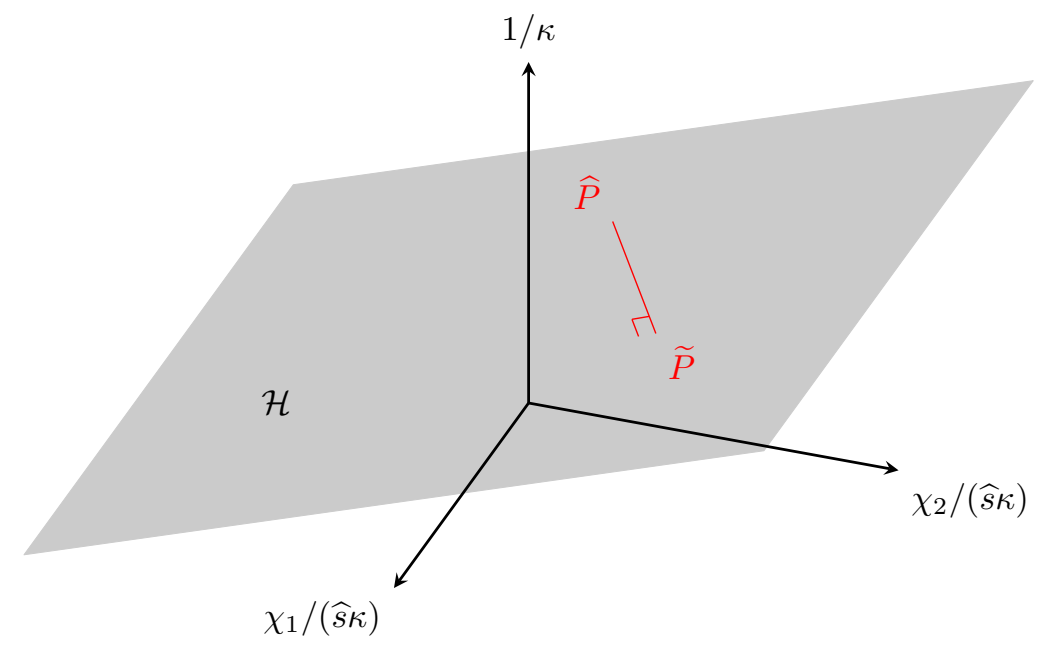

Figure 1: Illustration with 2 species of the projection $\widetilde{P}$ of the first approximation $\widehat{P}$ on the hyperplane $\mathcal{H}$ defined by 19

Depending on the gap magnitude between the left and right states, several approximate quadrature formulae can be used for evaluating integrals. We here privileged the Simpson rule, which is mainly adapted for large variation between two states. For the approximation of $\kappa$, this gives:

$$
\hat{\kappa}=\frac{1}{6}\left(\kappa_{L}+4 \kappa\left(\epsilon_{M}, \rho_{1, M}, \ldots, \rho_{n_{s}, M}\right)+\kappa_{R}\right),
$$

with $\epsilon_{M}=\frac{1}{2}\left(\epsilon_{L}+\epsilon_{R}\right)$ and $\rho_{i, M}=\frac{1}{2}\left(\rho_{i, L}+\rho_{i, R}\right)$

Finally, in order to satisfy the properties (iv) of the Roe solver, an orthogonal projection is carried out from the predicted values $\widehat{\kappa}$ and $\widehat{\chi}_{i}, i=1, \ldots, n_{s}$ on the $n_{s}-1$ hyperplan defined by the equation $19 \mathrm{p}$. In order to be independent of the arbitrary constant present in the definition of $\epsilon$ and to be close to a dimensionless orthonormal framework, we perform the orthogonal projection in the following system of coordinates:

$$
\left(\frac{1}{\kappa},\left\{\frac{\chi_{i}}{\widehat{s} \kappa}, i=1, \ldots, n_{s}\right\}\right)
$$

$\widehat{s}$ is a normalization factor with the dimension of $\chi_{i}$ chosen as $\widehat{s}=\widehat{c^{2}}=$ $\sum_{i=1}^{n_{s}} \widehat{\chi_{i} Y_{i}}+\widehat{\kappa h}$, where the symbol (.) corresponds in this last expression to the Simpson rule 22 . The configuration with two species where the hyperplane (19) corresponding to a $2 \mathrm{D}$ plane is illustrated Figure 1 .

The orthogonal projection in the space of coordinates $(23)$ from the predicted values $\widehat{\kappa}$ and $\widehat{\chi}_{i}, i=1, \ldots, n_{s}$ on the hyperplane defined by 19 gives the com- 
pressibility factors:

$$
\widetilde{\kappa}=\frac{D \hat{\kappa}}{D-\Delta p \delta p} \quad \tilde{\chi}_{i}=\frac{D \hat{\chi}_{i}+\hat{s}^{2} \Delta \rho_{i} \delta p}{D-\Delta p \delta p},
$$

with

$$
D=\hat{s}^{2} \sum_{i=1}^{N}\left(\Delta \rho_{i}^{2}\right)+\Delta p^{2},
$$

and the error on pressure $\delta p$ that, finally by construction, satisfies the precision up to machine accuracy after the projection step:

$$
\delta p=\Delta p-\hat{\kappa} \Delta \epsilon-\sum_{i=1}^{N} \hat{\chi}_{i} \Delta \rho_{i} .
$$

The celerity of sound at the Roe averaged state then becomes:

$$
\widetilde{c}_{s}=\sqrt{\sum_{i=1}^{n_{s}} \widetilde{\chi}_{i} \widetilde{Y}_{i}+\widetilde{\kappa} \tilde{h} .}
$$

This procedure allows us to obtain a combination of compressibility factors and an approximation of the sound speed that is coherent with the Roe properties at the intermediate state. The present procedure generalizes the Roe scheme for multi-component gas flow with an arbitrary convex equation of state.

It can be noted that the existence of $\widetilde{c}_{s}$ from (27) is not guaranteed since nothing constrains the variation of the compressibility factors to avoid the negative value of the sum inside the square root. However, this numerical scheme is robust enough in the cases studied here with non-calorically perfect gas. Indeed, the Simpson rule gives a quite precise estimation of the compressibility factors, so their variations are small during the projection. Moreover, the normalization factor $\widehat{s}$ brings the basis used for the projection 23 close to an orthonormal basis which prevents significant variation of one factor compared to the other during the orthogonal projection. The final value for the sound speed is then generally close to the initial left and right values. Some additional constraints may be necessary in very low density cases, but this aspect is not in the scope of this paper and therefore has not been investigated here.

\section{Numerical approach}

The resolution of the Euler equation (11) is based on a finite volume approach on a Cartesian grid. As pointed out previously, to recover a high-quality solution of the compressible multi-component flow, numerical approximations must satisfy three properties:

(i) a high-order accuracy in smooth regions of the solution;

(ii) solutions free from spurious oscillations around discontinuities;

(iii) a sharp resolution of discontinuities, which means there are only few cells within discontinuities (compare to low order simulation). 
To fulfill these three properties with multicomponent real gases, we continue the work done by Daru \& Tenaud [2, 18] in the development of high-order accurate schemes based on a Lax-Wendroff approach for unsteady shocked ideal gas flow predictions. We extend the proposed One-Step Monotonicity-Preserving (OSMP) scheme to tackle real gas flow prediction with modification of the total energy flux formulation. We also combined this scheme with an Adaptive Multiresolution procedure to automatically tighten grid points in regions where steep gradients occur.

Continuing the work done by Daru \& Tenaud [2, 18] in the development of highorder accurate schemes based on a Lax-Wendroff approach for unsteady shocked ideal gas flow predictions, we extend the One-Step Monotonicity-Preserving (OSMP) scheme able to tackle real gas flow predictions.

\subsection{One-step Monotonicity Preserving scheme}

In the following, we denote by $\mathbf{U}_{j}^{n}$ the discrete quantity $\mathbf{U}(\mathbf{x}, t)$ estimated at a grid point $\mathbf{x}_{j}=(j \delta \mathbf{x})^{\mathrm{T}}$ and at a time $t^{(n)}=n \delta t(\delta t, \delta \mathbf{x}$ being, respectively, the time step and the grid spacing and $j$ the index of the position in the particular dimension).

The Euler equations are discretized using a high-order one-step Monotonicity Preserving scheme, called the OSMP $p$ scheme 2, based on a Lax-Wendroff approach, which ensures a $p^{t h}$-order accuracy in both time and space in regular regions.

We first present the unlimited scheme on the one dimensional Euler equation:

$$
\frac{\partial \mathbf{U}}{\partial t}+\frac{\partial \mathbf{F}(\mathbf{U})}{\partial x}=\mathbf{0}
$$

which is discretized using the following conservative approximation:

$$
\mathbf{U}_{j}^{n+1}=\mathbf{U}_{j}^{n}-\frac{\delta t}{\delta x}\left(\widetilde{\mathbf{F}}_{j+1 / 2}-\widetilde{\mathbf{F}}_{j-1 / 2}\right) .
$$

The numerical flux $F_{j+1 / 2}$ is approximated by a $p^{t h}$-order One-Step scheme $(\mathrm{OS} p)$ developed in [2]:

$$
\widetilde{\mathbf{F}}_{j+1 / 2}=\widetilde{\mathbf{F}}_{j+1 / 2}^{R o e}+\frac{1}{2} \sum_{k=1}^{N}\left(\Phi_{k}^{p}\left(1-\left|\nu_{k}\right|\right) \widetilde{\alpha}_{k}\left|\widetilde{\lambda}_{k}\right| \cdot \widetilde{\mathbf{K}}^{(k)}\right)_{j+1 / 2}
$$

where $\widetilde{\mathbf{F}}_{j+1 / 2}^{R o e}$ is the Roe flux at the cell interface given by 10 following the Roe solver expressed in the previous section $\left(\S 3.1\right.$. $\nu_{k, j+1 / 2}=\frac{\delta t}{\delta x} \widetilde{\lambda}_{k, j+1 / 2}$ is the local CFL number evaluated at cell interfaces.

$\Phi_{k_{j+1 / 2}}^{p}$ are the $p^{t h}$-order accuracy functions that are split into even and odd contributions corresponding to respectively, even derivatives, expressed using centered approximations, and odd derivatives that are based on upwind ap- 
proximations. They can be found in [2], and are recalled here for completeness:

$$
\Phi_{k_{j+1 / 2}}^{p}=\sum_{n=1}^{m} \Psi_{k_{j+1 / 2}}^{2 n}-j s \sum_{n=1}^{m_{1}} \Psi_{k_{j+1 / 2-j s / 2}^{2 n+1}}^{2 n}
$$

where $m=\lfloor p / 2\rfloor, m_{1}=\left\lfloor\frac{(p-1)}{2}\right\rfloor(\lfloor\rfloor$ is the integer division symbol), and $j s=\operatorname{sign}\left(\widetilde{\lambda}_{k, j+1 / 2}\right)$. The even and odd functions $\Psi_{k j+1 / 2}^{2 n}$ and $\Psi_{k_{j+1 / 2-j s / 2}^{2 n+1}}^{2 n}$ are given by the recurrence formula (for $n \geq 1$ ):

$$
\begin{aligned}
\Psi_{k j+1 / 2}^{2 n} & =\sum_{l=0}^{2 n-2}(-1)^{l} C_{2 n-2}^{l} \cdot\left(c_{k}^{(2 n)} \widetilde{\alpha}_{k}\right)_{j+1 / 2+n-1-l}, \\
\Psi_{k+1 / 2}^{2 n+1} & =\sum_{l=0}^{2 n-1}(-1)^{l} C_{2 n-1}^{l} \cdot\left(c_{k}^{(2 n+1)} \widetilde{\alpha}_{k}\right)_{j+1 / 2+(n-1-l) . j s} ;
\end{aligned}
$$

where $C_{r}^{l}=\frac{r !}{(r-s) ! s !}$.

The coefficients $c_{k}{ }^{(q)}$ depend on the local CFL number $\left(\nu_{k j+1 / 2}\right)$, and are given by:

$$
\left(c_{k}^{(q+1)}\right)_{j+1 / 2}=\frac{\left|\nu_{k}\right|_{j+1 / 2}+(-1)^{q}\left\lfloor\frac{(q+1)}{2}\right\rfloor}{q+1} \cdot\left(c_{k}^{(q)}\right)_{j+1 / 2}, \quad q \geqslant 2,
$$

with

$$
c_{k_{j+1 / 2}^{(2)}}^{(2)}=\left|\lambda_{k}\right|_{j+1 / 2}\left(1-\left|\nu_{k}\right|_{j+1 / 2}\right) .
$$

Using the accuracy function $\Phi^{p}$ (31), the scheme is $p^{\text {th }}$-order accurate in both space and time by using the solution at only $p+2$ grid cells.

To overcome the drawback of spurious oscillations in the vicinity of steep gradient, we employ Monotonicity-Preserving (MP) constraints that locally relax the TVD constraints near extrema. These constraints have been first developed by Suresh and Huynh [19] and further extended by Daru and Tenaud [2]. As, for each $k$-wave, accuracy functions $\Phi_{k}^{p}$ are based on the Riemann invariants $\widetilde{\alpha}_{k}$, the numerical flux at cell interfaces can be recast into:

$$
\widetilde{\mathbf{F}}_{j+1 / 2}=\frac{1}{2}\left(\mathbf{F}_{j+1}+\mathbf{F}_{j}\right)-\frac{1}{2} \sum_{k=1}^{N}\left(\widetilde{\alpha}_{k}^{M-P}\left|\widetilde{\lambda}_{k}\right| \cdot \widetilde{\mathbf{K}}^{(k)}\right)_{j+1 / 2},
$$

where $\widetilde{\alpha}_{k}^{M-P}$ are function of the Riemann invariants:

$$
\widetilde{\alpha}_{k}^{M-P}=\left[\left(1-\Phi_{k}^{p M-P}\left(1-\left|\nu_{k}\right|\right)\right) \widetilde{\alpha}_{k}\right]_{j+1 / 2}, k \in[1, N] .
$$

Daru and Tenaud 2] proposed to apply the M-P constraints for each $k$-wave on the accuracy functions $\left(\Phi_{k}^{p}\right)$ to recover a scheme that is $p^{t h}$-order in time and space everywhere except near discontinuities where the scheme is MonotonicityPreserving. 
In the following, accuracy functions of the $7^{\text {th }}$-order that can be found in Daru \& Tenaud [2], have been used for computing test-cases.

The extension in the multidimensional case is delicate as far as a coupled time and space approach is used since cross derivative terms that appear in the second and higher-order terms are left uncontrolled if one applies a direction by direction MP correction to a Lax-Wendroff unsplit scheme. We also need to guarantee that the resulting scheme is non-oscillatory. The simplest way to avoid solving cross derivatives and to recover good properties of the onedimensional scheme is to use a Strang directional splitting strategy [20, 21]. While the order of accuracy is lowered compared to the tensorial multistage approach, the OSMP scheme with the Strang algorithm provides results with a very small error level at low-cost [2, 18].

In two dimensions, the splitting of the system of equations can be constructed to give an accurate symmetric solution every two time-steps:

$$
\mathbf{U}_{j}^{n+2}=\left(L_{\delta x}(\delta t) \cdot L_{\delta y}(\delta t)\right) \cdot\left(L_{\delta y}(\delta t) \cdot L_{\delta x}(\delta t)\right) \cdot \mathbf{U}_{j}^{n} .
$$

Here $L_{\delta x}$, and $L_{\delta y}$ are discrete approximations of the Euler operators in each space direction. For instance, $L_{\delta x}$ denotes the Euler operator of the following problem:

$$
L_{\delta x}(\delta t) \cdot\left(\mathbf{U}_{j}^{m}\right)=\mathbf{U}_{j}^{m}-\frac{\delta t}{\delta x}\left(\mathbf{F}_{j+1 / 2}^{m}-\mathbf{F}_{j-1 / 2}^{m}\right) .
$$

In three dimensions, the splitting operator arrangement can be found in [22].

\subsection{Proposed modification of the total energy flux formulation for the general- ization of the multi-component real gas Roe solver}

In $\S 3.3$, we introduced how to calculate an averaged state of the compressibility factors that respects the properties of the Jacobian matrix of the Euler flux. This is carried out through an orthogonal projection on the $n_{s}-1$ hyperplane defined by (19). However, through the use of several test cases, numerical artifacts close to discontinuities and poor order of convergence may be observed (some examples will be provided in the section devoted to results; see $\S 5$.

Indeed in some configurations, despite the orthogonal projection procedure, there is an infinite number of solutions that respect the Roe solver properties, especially when the dimension of the problem is high in multicomponent. Thus, most of the time, the Roe averaged state obtained does not necessarily correspond to a realistic thermodynamic state. This problem does not appear when a first-order scheme, i.e. the original Roe scheme, for instance (10), is used, but this drawback only occurs when a high-order scheme is employed because of the large extent of the stencil. In fact, when a wide stencil is required, the evolution of the averaged compressibility factors can become inconsistent with the evolution of the mass fraction. Thus, inaccurate approximation of the compressibility factors at the Roe average state can reduce the effective order of convergence of the scheme around a large variety of composition and/or thermodynamic variables in a smooth solution. In the same way, numerical artifacts are also introduced when significant variations of species occur in the vicinity of contact discontinuities. 
By analyzing the problem, we found that it is coming from the relationship used to reconstruct the numerical flux related to the total energy 110 . The ratio of compressibility factors explicitly appears through a linear combination of the Riemann invariants associated to the same eigenvalue $u$ attributed to contact waves:

$$
\begin{aligned}
\Delta F_{\rho E}=\Delta(\rho u H)= & \sum_{i=1}^{n_{s}} \widetilde{\alpha}_{i}\left(\widetilde{E_{c}}-\frac{\widetilde{\chi}_{i}}{\widetilde{\kappa}}\right)|\widetilde{u}|+\widetilde{\alpha}_{N-1}\left(\widetilde{H}+\widetilde{u} \widetilde{c}_{s}\right)\left|\widetilde{u}+\widetilde{c}_{s}\right| \\
& +\widetilde{\alpha}_{N}\left(\widetilde{H}-\widetilde{u} \widetilde{c}_{s}\right)\left|\widetilde{u}-\widetilde{c}_{s}\right|
\end{aligned}
$$

Considering the definition of the speed of sound, given by (27), and the relationship 19 linking the pressure jump to the density and the internal energy jumps through the compressibility factors, the first term of the total energy flux (39), noted $\widetilde{\alpha}_{N+1}$, can be expressed using variations of several variables:

$$
\widetilde{\alpha}_{N+1}=\sum_{i=1}^{n_{s}} \widetilde{\alpha}_{i}\left(\widetilde{E_{c}}-\frac{\widetilde{\chi}_{i}}{\widetilde{\kappa}}\right)=\Delta(\rho E)-\widetilde{H} \frac{\Delta p}{\widetilde{c}_{s}^{2}}-\widetilde{\rho} \widetilde{u} \Delta u,
$$

Let us note that the proposed term $\left(\widetilde{\alpha}_{N+1}\right)$ follows an advection equation similarly to the classical Riemann invariants $\left(\widetilde{\alpha}_{k}, k \in[1, N]\right)$. This additional advection equation is then solved using a specific accuracy function $\Phi_{\alpha_{N+1}}^{p}$ as described in $\S 4.1$. Then, the total energy flux is expressed without being explicitly connected to the independent values of the compressibility factors but only using the value of the sound speed obtained from the combination (27). We then apply an MP condition on the supplementary accuracy function $\Phi_{\alpha_{N+1}}^{p}$ to avoid spurious oscillations. The total energy component of the high-order M-P limited Euler flux then reads:

$$
\begin{aligned}
\widetilde{F}_{\rho E}^{p-M P}=\frac{1}{2}\left(F_{L}(\rho E)+F_{R}(\rho E)\right) & -\frac{1}{2}\left(\widetilde{\alpha}_{N+1}^{M-P}|\widetilde{u}|+\widetilde{\alpha}_{N-1}^{M-P}\left(\widetilde{H}+\widetilde{u} \widetilde{c}_{s}\right)\left|\widetilde{u}+\widetilde{c}_{s}\right|+\right. \\
& \left.\widetilde{\alpha}_{N}^{M-P}\left(\widetilde{H}-\widetilde{u} \widetilde{c}_{s}\right)\left|\widetilde{u}-\widetilde{c}_{s}\right|\right),
\end{aligned}
$$

with $\widetilde{\alpha}_{N-1}$, and $\widetilde{\alpha}_{N}$ given by 37 .

From a mathematical point of view, applying the OSMP scheme on $\widetilde{\alpha}_{N+1}$ independently of the $n_{s}$ first $\widetilde{\alpha}_{i}$ is actually equivalent to define a constraint on the compressibility factors which results in corrected effective values $\overline{\bar{\chi}}_{i}, i=1, \ldots, n_{s}$ and $\overline{\bar{\kappa}}$ that are solutions of the set of equations:

$$
\left\{\begin{array}{l}
\Delta p=\sum_{i=1}^{n_{s}} \overline{\bar{\chi}}_{i, j+1 / 2} \Delta \rho_{i}+\overline{\bar{\kappa}}_{j+1 / 2} \Delta \epsilon \\
\widetilde{c}_{s, j+1 / 2}^{2}=\sum_{i=1}^{n_{s}} \overline{\bar{\chi}}_{i, j+1 / 2} \widetilde{Y}_{i, j+1 / 2}+\overline{\bar{\kappa}}_{j+1 / 2} \widetilde{h}_{j+1 / 2} ; \\
\widetilde{\alpha}_{N+1, j+1 / 2}^{M-P}=\sum_{i=1}^{n_{s}} \widetilde{\alpha}_{i, j+1 / 2}^{M-P}\left(\widetilde{E}_{c j+1 / 2}-\frac{\overline{\bar{\chi}}_{i, j+1 / 2}}{\overline{\bar{\kappa}}_{j+1 / 2}}\right) .
\end{array}\right.
$$

The first two relationships correspond to the properties that must be preserved 
to fulfill the jump relationships of the Riemann problem and the definition of the sound speed predicted by our proposed extension of the Vinokur and Montagné algorithm (see $\S 3.3$. The third one corresponds to the relation that must be verified once applied the OSMP scheme on the additional Riemann invariant $\widetilde{\alpha}_{N+1}$. Since the expression of the flux 41 no longer requires explicit values for the compressibility factors, then we have to guarantee the existence of the solution.

Equations 42 correspond to a linear system with $n_{s}+1$ unknowns and three equations. The existence of a set of compressibility factors consistent with the Roe solver properties and the $N+1$ accuracy functions and their associated $\mathrm{M}-\mathrm{P}$ constraints is then guaranteed. In the case of two species, there is even a unique solution corresponding to the sound speed value previously predicted.

That way, we thus obtain a stable high-order scheme based on a Roe solver for multi-component real gases that preserves conservation through jump relations and properly capture discontinuities on the mass fraction of species. An overview of the different steps leading to the final high-order Euler flux is proposed in the following algorithm 2

\subsection{Adaptive Multiresolution procedure for grid refinement}

Compressible multi-component flows are known to exhibit spatial multi-scales that need to be resolved in the DNS approach. That is why we here employ a grid refinement technique to tighten grid points in regions where physical phenomena occur and coarsen them elsewhere. As we stressed previously, simulations of compressible multi-component flow need to use an accurate numerical scheme coupled with refined grids in regions where physical phenomena occur. We then employed an Adaptive Multiresolution procedure to automatically refine the grid locally in regions where steep gradients as well as unsteady flow phenomena occur and save grid points elsewhere. To be combined with high-order schemes, the mesh refinement must be based on a technique that provides error estimates to control the solution error, and we based our grid refinement strategy on a Multiresolution Analysis (MRA), originally introduced by A. Harten[23], and theoretically developed by A. Cohen and co-authors $[24,3$.

As we follow a finite volume approach, the cell-averaged multiresolution analysis [23 has been employed. In the context of adaptive mesh refinement, the multiresolution analysis represents data on a set of nested dyadic grids. A tree data structure is used to generate embedded grids and to encode the multiresolution analysis procedure. A projection operator allows computing the cell-average solution at a coarse grid level, knowing cell-average values at a finer grid level. This projection operator is exact and unique. A prediction operator maps the cellaverage solution at a fine grid level to an approximation at a coarser grid level, using centered linear polynomial interpolation that is chosen consistent with the projection operator to satisfy conservation [24]. The refinement criterion is based on a measure of details that are prediction error estimates, calculated with the difference between the known numerical solution at a grid level and its interpolated value at the same grid level. If the measure of details in a cell is less than a threshold value (namely $\sigma$ ), this one is discarded from the tree data structure, and the tree is pruned. Harten heuristic criterion ([25] for details) 


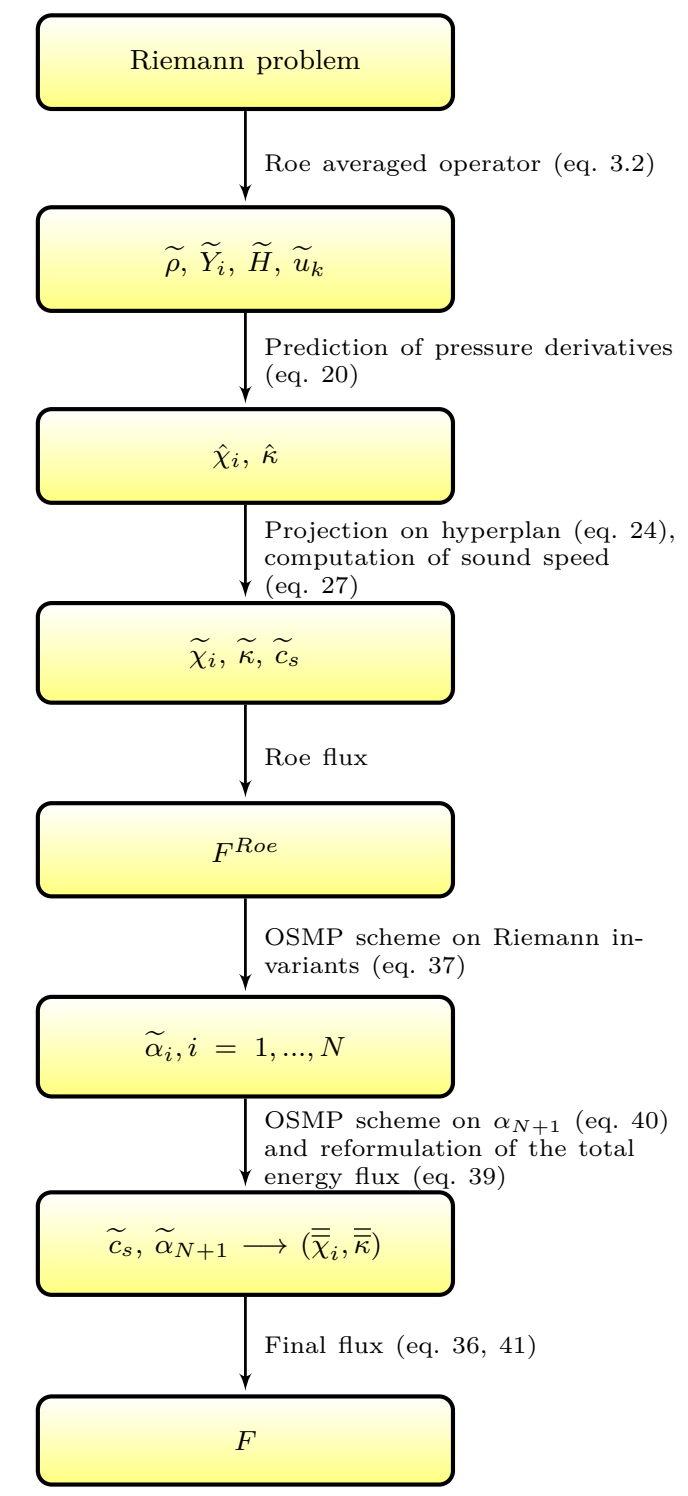

Figure 2: Successive steps for building a stable OSMP scheme for multi-component real gas flow 
also based on the measure of details is employed to refine the mesh when necessary. In the following results, the threshold is set at a classical value: $\sigma=10^{-3}$. We do not further describe the method since the principle of MRA coupled with OSMP scheme has already been presented in detail in Tenaud et al. 25. In this work, capabilities to recover elementary physical mechanisms of the MRA coupled with OSMP scheme have been assessed, showing that the solution quality has been clearly improved with significant gains in both CPU time and memory usage, compared to single grid computations. However, let us add that if consecutive cells have not the same grid refinement, the numerical flux at the cell interface has been evaluated at the highest grid refinement between these two consecutive cells, using adjacent virtual cells. A special attention is devoted to calculating ingoing and outgoing fluxes at an interface between consecutive cells at different grid levels to ensure strict conservation of fluxes at this interface. See 25] for more details.

\section{Numerical results}

\subsection{Mixture of non-calorically perfect gas with temperature-dependent heat ca- pacities}

In the following simulations, mixtures of non-calorically perfect gases are considered. The general gas equation is used:

$$
p=\rho r T
$$

with $r=r\left(Y_{1}, \ldots, Y_{n_{s}}\right)$ the constant of the mixture of gases. To reproduce the behavior of real gases, vibrational degrees of freedom of polyatomic molecules are taken into account by considering the dependency of the specific heat capacities of the species with respect to the temperature.

$$
d e=c_{v}(T) d T \quad d h=c_{p}(T) d T
$$

Temperature polynomials are used to approximate the value of the heat capacities of the species. Coefficients of these polynomials are available in Appendix $\mathrm{D}$ of 26 . This formalism induces that the heat capacity ratio $\gamma$ and the compressibility factors are not constant and depend on the temperature and the composition of the mixture. It implies that the classical expression of the Roe average is no more valid for this thermodynamic model because compressibility factors are involved in the Jacobian matrix 9. To illustrate the temperature dependence, we plot in Figure 3 the heat capacities at constant pressure versus the temperature for the three species involved (N2, O2, and R22) in the following simulations.

While significant variations with the temperature are clearly recorded, let us notice that $C p_{R 22}$ shows a drastic change in the range of temperature $T \in$ $[300 ., 1000$.$] , leading to relevant changes of the heat capacity ratio \left(\gamma_{R 22}\right)$ as well as the speed of sound $\left(c_{R 22}\right)$.

We noticed that the coefficients of the polynomes allow us to keep a convex hyperbolic system [27] and preserve the assumption made on the equation of state (3). 


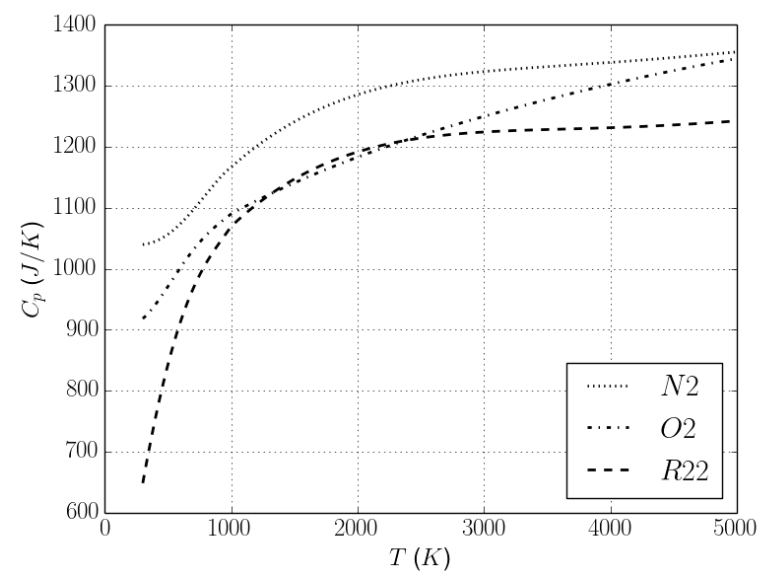

Figure 3: Distribution versus temperature of heat capacities at constant pressure $(\mathrm{Cp})$ following temperature polynomials found in [26] for Nitrogen (N2), Oxygen (O2), and R22.

\subsection{Convection of mass fraction and density waves}

This first case performs a grid-convergence analysis. The high-order scheme is applied on a smooth case of convection of mass fraction and density waves in a flow with uniform pressure. This case has previously been treated in [28]. We consider a mass fraction wave in a flow with uniform velocity $u_{0}=100 \mathrm{~m} / \mathrm{s}$ and pressure $p_{0}=1$ Bar. The mixture is composed of two gases with specific heat ratio of $\gamma_{1}=1.6$ and $\gamma_{2}=1.4$. The specific isochore heat capacity is set in order to have $C v_{1}=2 C v_{2}$. The computational domain corresponds to $\Omega=[0,1]$ with periodic conditions. The initial mass fraction of the first species and the initial density are:

$$
Y_{1}(x)=\frac{1}{2}+\frac{1}{4} \sin (4 \pi x), \quad \rho_{0}(x)=1+\frac{1}{2} \sin (2 \pi x) \forall x \in \Omega
$$

Computation is performed using the OSMP scheme of $7^{\text {th }}$-order with and without the modification on the discrete total energy flux using the $\alpha_{N+1}$ term described in 40 and $(41)$. The exact solution is the passive convection of the waves. Errors in the $L_{1}$-norm relative to the exact solution are reported in Figure 4 at $t=0.05 \mathrm{~s}$ after five periods of convection.

It is found that in this case, with significant variations in composition and temperature leading to relevant variation of sound speed, the initial estimation of the compressibility factors with the extension of Vinokur and Montagné method described in $\S 3.3$ provides a scheme which is only second-order accurate. Their successive values are effectively inadequate with the physical mixture and pressure variations are generated, impacting the flow velocity and the density profile. The introduction of $\widetilde{\alpha}_{N+1}$ in the first term of the total energy flux 39 avoids the independent influence of the different compressibility factors and allows to recover the seventh-order accuracy of the OSMP scheme. We also noticed that the M-P condition does not influence results in this smooth test case except in low resolution with only 32 grid points by differentiating extrema from discontinuities. 


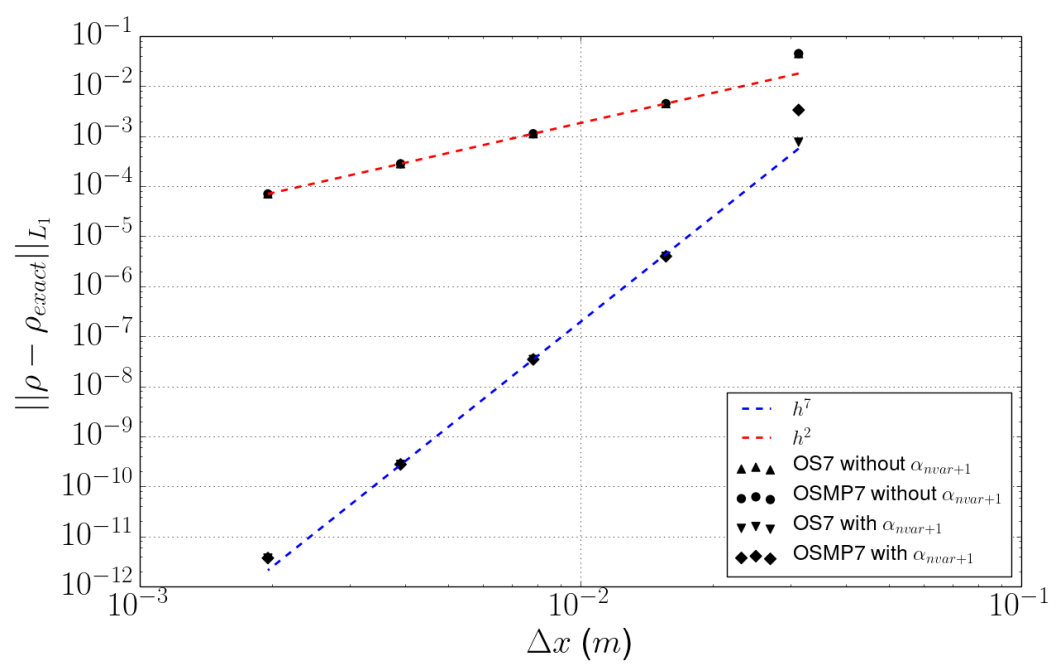

Figure 4: Convergence curves using the OSMP7 scheme on a advection of mass fraction wave. Comparison with the initial formulation of the total energy flux and the new one with and without $M-P$ limiter flux.

\subsection{One-dimensional shock-tube problem}

We consider classical cases of shock tube problems corresponding to exact Riemann problems. The tube length is $L_{x}=50 \mathrm{~m}$, and the computational domain extent is $x \in\left[0, L_{x}\right]$. We initially consider two states of different mixtures, separated by a diaphragm located at the middle of the tube $(x=25 \mathrm{~m})$. At the initial time $(t=0)$, the diaphragm is broken. Classically, a shock moves towards the low-pressure side while a rarefaction fan goes to the high-pressure side. In between, a contact discontinuity associated with the three species moves at the fluid velocity.

The initial conditions at the left state $\mathcal{U}_{L}$ and right state $\mathcal{U}_{R}$ are given in table 1 The first one corresponds to the classical Sod shock tube test case and the second corresponds to the Lax shock tube test case that is generally subject to more spurious oscillations. Each mixture is composed of three species, the species $\mathrm{O}_{2}$ stays constant across the shock wave. The use of three species is significant to test the robustness of our method because this additional dimension increases the possible solutions at the Roe intermediate state. A constant value sensitive to the numerical error with high-order simulation illustrates the accuracy of the estimation and the correction around the contact wave.

In the following, simulations are performed with 640 grid cells with a CFL number $C F L=0.9$. No adaptive refinement is used on those cases to evaluate only the effects of the MP correction. The final times are chosen so that extreme waves do not have time to reach the domain boundaries and keep the values of conservative variables unchanged at the computational domain limits.

Results of the Sod shock tube obtained after $30 \mathrm{~ms}$ are presented in Figure 5 . The solution obtained with the proposed corrected high-order OSMP scheme is compared to the original (uncorrected) OSMP solution and the first-order Roe solver. As usual, these distributions clearly show, from right to left, the 


\begin{tabular}{lcccc}
\hline & \multicolumn{2}{c}{ Sod shock tube } & \multicolumn{2}{c}{ Lax shock tube } \\
\hline & Left & Right & Left & Right \\
\hline $\mathrm{N}_{2}(\%)$ & 70 & 20 & 70 & 20 \\
$\mathrm{R}_{22}(\%)$ & 20 & 70 & 20 & 70 \\
$\mathrm{O}_{2}(\%)$ & 10 & 10 & 10 & 10 \\
$P(\mathrm{bar})$ & 1 & 0.1 & 10 & 1 \\
$\rho\left(\mathrm{kg} / \mathrm{m}^{3}\right)$ & 1. & 0.125 & 1.602 & 1.222 \\
$\gamma$ & 1.35 & 1.20 & 1.26 & 1.19 \\
\hline
\end{tabular}

Table 1: Initial condition of pressure, density and species mass fraction for the Sod shock tube (left) and the Lax shock tube (right) problems

shock wave, the contact discontinuity and the rarefaction fan, separated with constant solution values. The use of a high-order scheme with a monotonicity preserving feature significantly reduces the numerical diffusion and more properly captures discontinuities and rarefaction waves compared to a classic first-order Roe solver, as illustrated in Figure 5 However, when the original (uncorrected) OSMP scheme is applied, numerical artifacts appear in the contact discontinuity mainly due to the evaluation of compressibility factors that do not necessarily correspond to a realistic thermodynamic state. Using the additional M-P criteria on the combination of Riemann invariants (37), the resulting intermediary state is closer to a physically realistic state, and the mass fraction contact discontinuity is captured with robustness (see Figure 5).

To validate the solution obtained with the proposed modified OSMP scheme, we compare it (Figure 6) to the exact solution of the Riemann problem obtained with the same thermodynamic data. These solutions are also compared to the exact solution with constant heat capacities for each species corresponding to the ideal gas problem. Thus, we could judge the high quality of the real gas solution obtained since it perfectly fits the exact solution. Discrepancies are clearly registered with the exact solution for an ideal gas, with differences in the wave speeds and the intermediary states between these waves. These discrepancies are coming from the variation of heat capacities of the species and then the difference of predicted speed of sound with the real gas treatment. These are mainly visible in the high-temperature region where the thermodynamics coefficient for R22 exhibits drastic variations (See Figure 3). These results justify the proposed approach for real gas flow predictions.

Similar results for the Lax shock tube are presented after $15 \mathrm{~ms}$ in Figure 7. A comparison with and without the corrected OSMP scheme illustrates the method's efficiency in reducing numerical artifact and capturing contact waves with minimal numerical diffusion. We noticed that pressure and velocity are constant within the contact discontinuity.

\subsection{The 2D test case: a shock wave interacting with a R22 bubble}

The interaction between a shock wave propagating in the air with a bubble of high-density gas is a classic test case often used to validate numerical approaches. It presents elementary physical mechanisms as the production of acoustic waves and vorticity through the baroclinic process. This test case, first introduced experimentally by J.-F. Haas [29, consists of a cylindrical bubble 

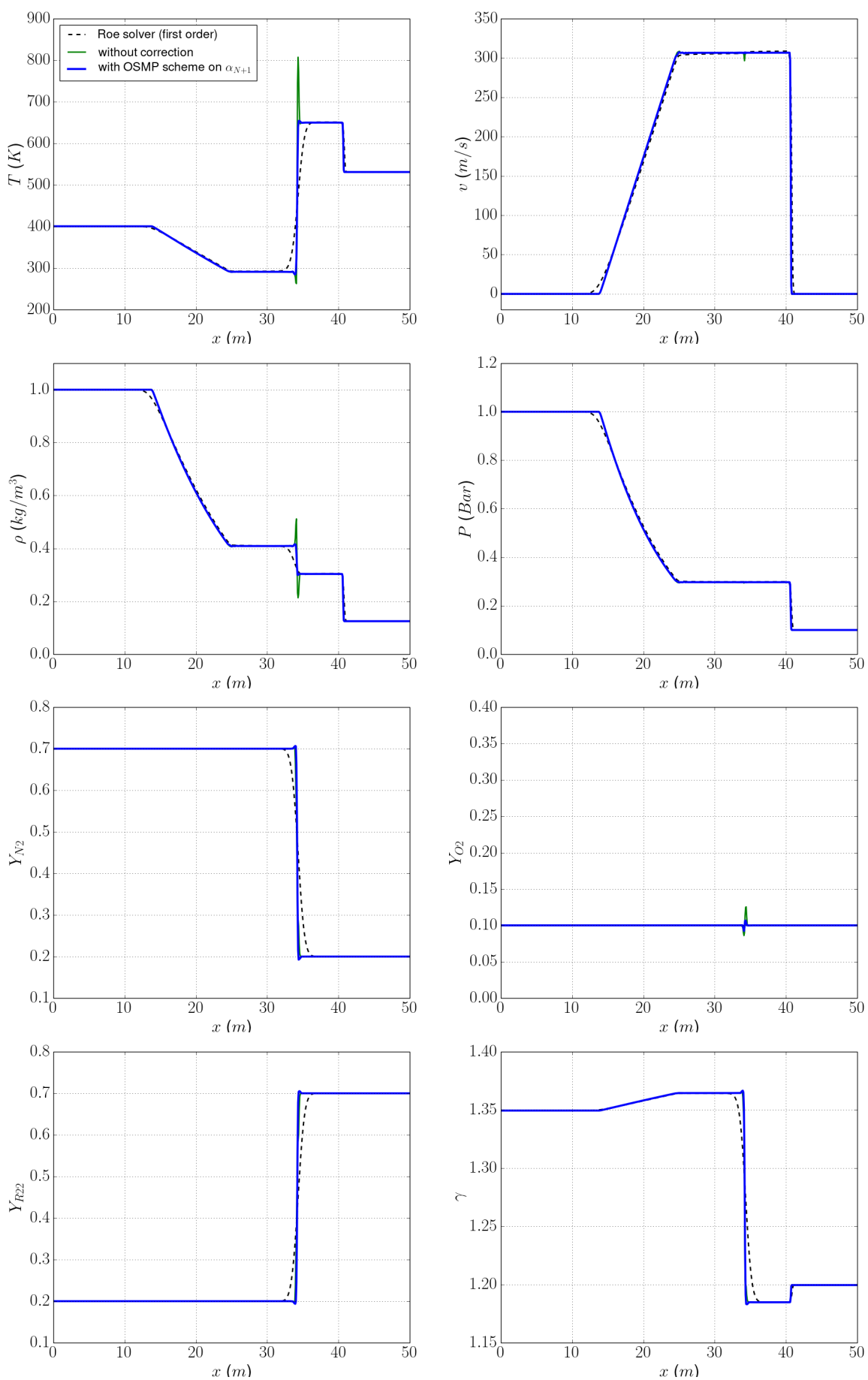

Figure 5: Distribution of the temperature, the velocity, the density, the pressure, the mass fraction of $\mathrm{N}_{2}, \mathrm{O}_{2}$ and $\mathrm{R} 22$ and the heat capacity ratio $\gamma$ for a $1 \mathrm{D}$ Sod shock tube at $t=30$ ms. Comparisons between the proposed corrected high-order OSMP scheme (bold blue line) with the original (uncorrected) OSMP scheme (bold green line) and the first-order Roe solver (black dashed line). 

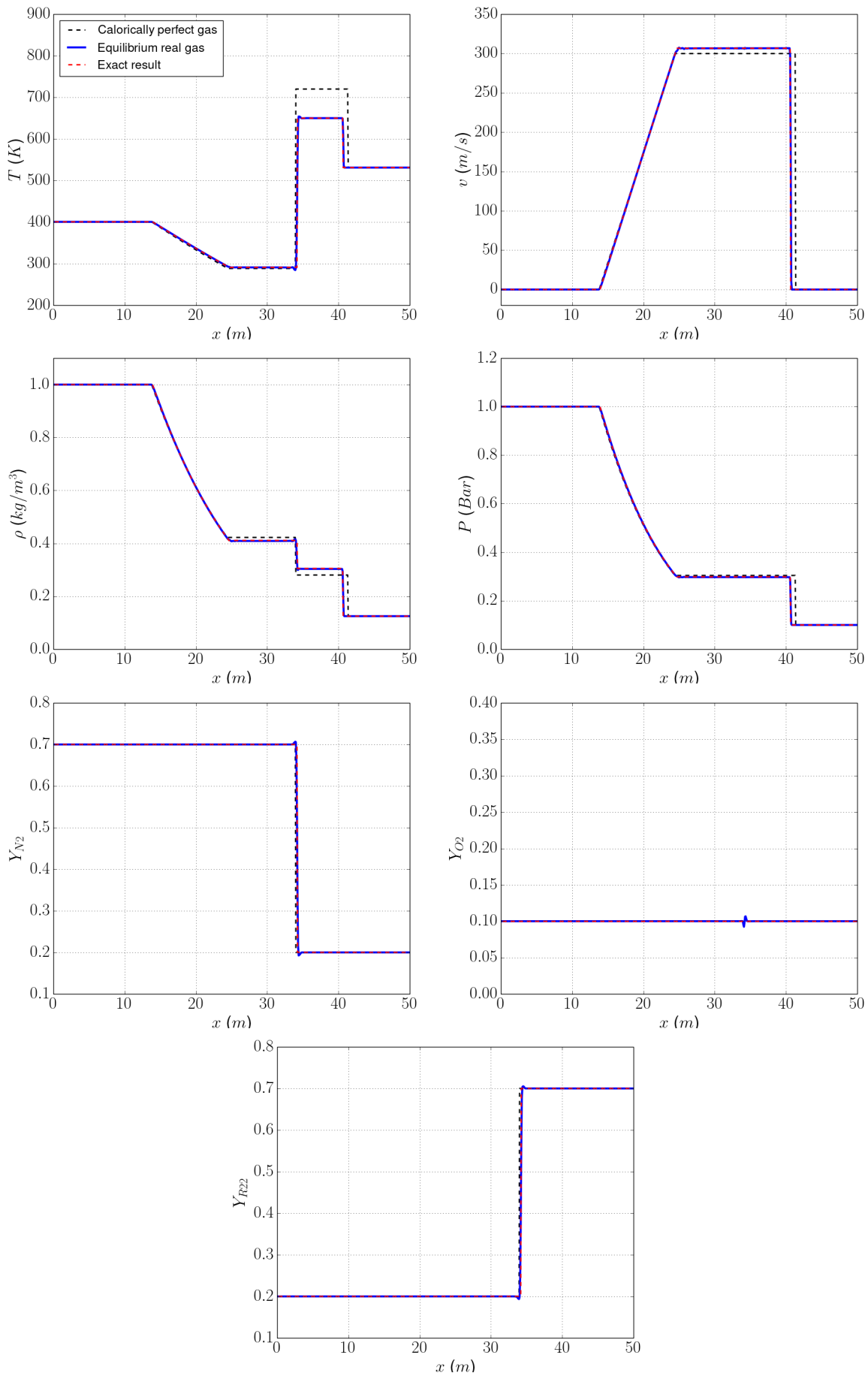

Figure 6: Comparison of the temperature, the velocity, the density, the pressure, and the mass fraction distributions for the $1 \mathrm{D}$ Sode shock tube at $t=30 \mathrm{~ms}$. Comparisons between results using calorically perfect gas (black dashed line) and Equilibrium real gas (bold blue line) with the exact solution obtained for real gases (red dashed line). 

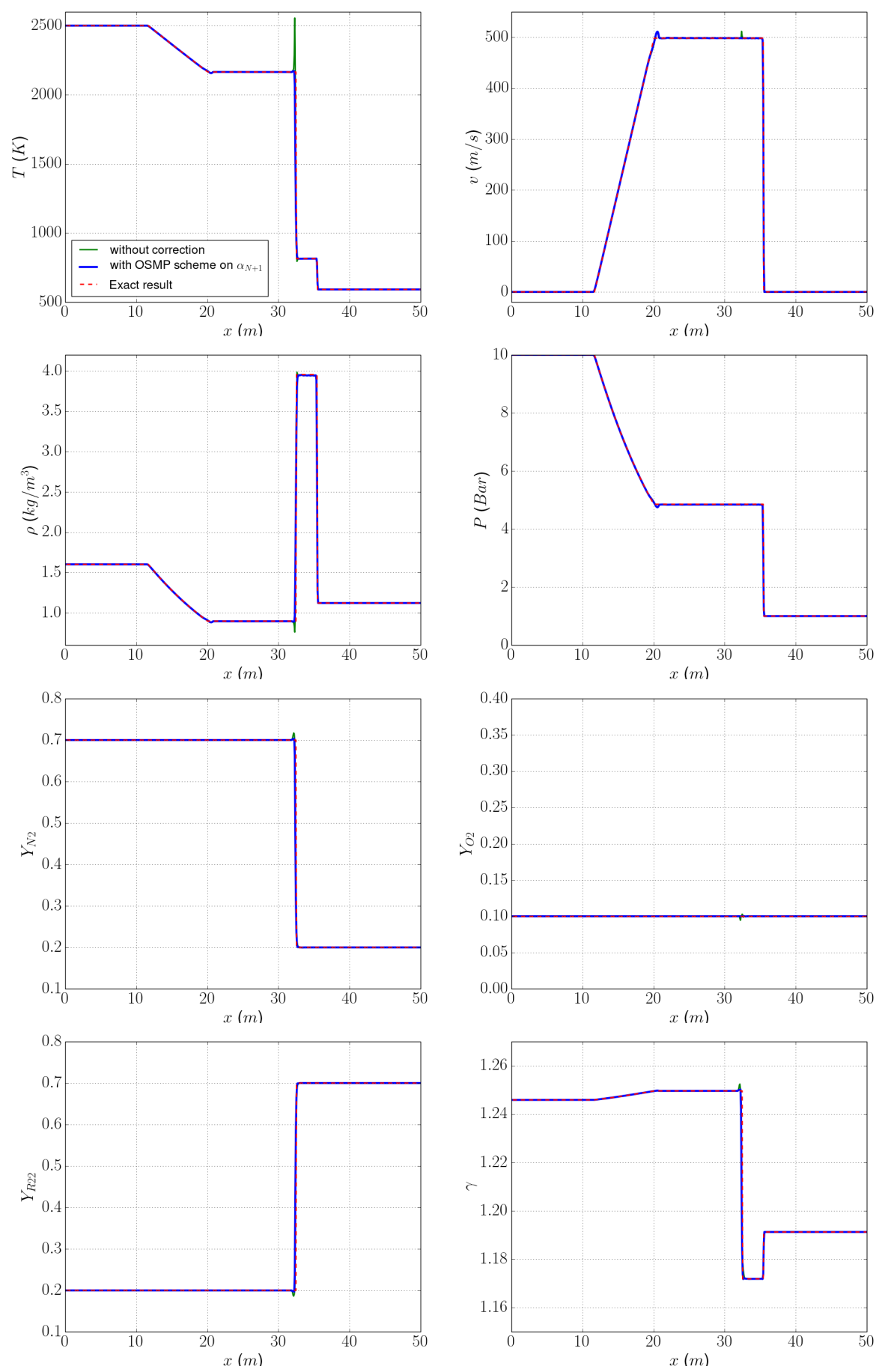

Figure 7: Comparison of the temperature, the velocity, the density, the pressure, the mass fraction, and specific heat ratio distributions for the $1 D$ Lax shock tube at $t=15$ ms, obtained with (bold blue line) and without (green line) the modified OSMP scheme and with the exact solution however for ideal gases (black dashed line). 


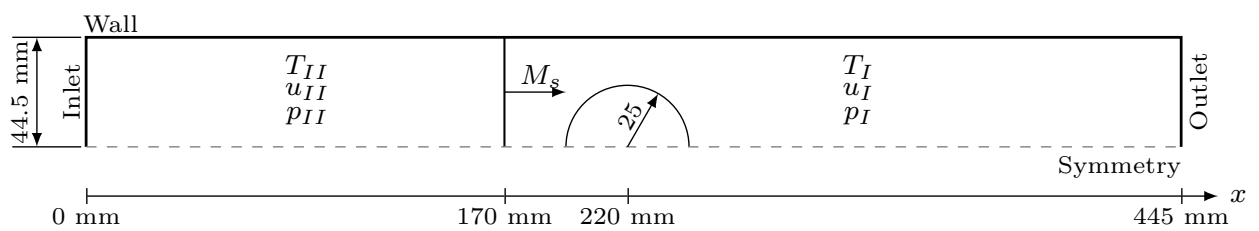

Figure 8: Sketch of the computational domain: initial configuration and boundary conditions.

filled with a dense hydrochlorofluorocarbon refrigerant R22 gas, initially located in ambient air at atmospheric pressure. A planar shock wave moving in ambient air at a Mach number $M_{s}=1.22$ towards the R22 bubble interacts with it. In the past, numerous numerical studies tackled this test case until recently with studies from [30] and 31].

The sketch of the initial configuration is given in Figure 8 where the computational domain is also illustrated. Only the upper half of the experimental configuration is simulated. A symmetric boundary condition is applied at the lower bound of the domain. At inlet and outlet boundaries, non-reflecting boundary conditions based on the work of Poinsot and Lele [32] are prescribed, although extreme waves do not reach these boundaries at the final simulation time. A solid wall is located at the top boundary, where slip condition is prescribed $\left(\mathbf{u} \cdot \mathbf{n}_{\text {wall }}=0\right.$, with $\mathbf{n}_{\text {wall }}$ the wall normal vector $)$ as well as symmetry condition for scalars.

The computational domain length is $L_{x}=445 \times 10^{-3} \mathrm{~m}$ and its height is $L_{y}=44.5 \times 10^{-3} \mathrm{~m}$. The R22 cylindrical bubble which initially has a diameter of $d_{0}=25 \times 10^{-3} \mathrm{~m}$, is initially centered on the bottom symmetry line at $x=220 \times 10^{-3} \mathrm{~m}$ from the inlet. The shock wave is initially located at $x=$ $170 \times 10^{-3} \mathrm{~m}$, and the state in front of the moving shock wave (noted region I, Figure 8D is prescribed to be at the atmospheric conditions with $T_{I}=298 \mathrm{~K}$, and $P_{I}=101325 \mathrm{~Pa}$. The fluid is initially at rest, so we have $u_{I}=0 \mathrm{~m} . \mathrm{s}^{-1}$

In the case of an ideal gas, Air is taken to have a heat capacity ratio of $\gamma_{0, \text { Air }}=$ 1.4 , and a heat capacity ratio of $\gamma_{0, R 22}=1.249$ for the dense R22 gas, which correspond, respectively, to heat capacity for Air of $C p_{A i r}=1005.5 \mathrm{~J}_{\mathrm{Jg}} \mathrm{kg}^{-1} . \mathrm{K}^{-1}$ and $C p_{R 22}=455.89 \mathrm{~J} \cdot \mathrm{kg}^{-1} \cdot \mathrm{K}^{-1}$ for R22. With a moving shock wave at $M_{s}=$ 1.22 , the post-shock conditions (region noted II) become $T_{I I}=339.88 \mathrm{~K}, u_{I I}=$ $115.64 \mathrm{~m} / \mathrm{s}$ and $p_{I I}=159060 \mathrm{~Pa}$.

Simulations was also performed for a real gas configuration with a mixture of $79 \%$ of $\mathrm{N}_{2}$ and $21 \%$ of $\mathrm{O}_{2}$ in Air leading to, respectively, mass fraction $Y_{N 2}=0.767$ and $Y_{O 2}=0.233$, while the bubble is filled with pure R22 dense gas. In this real gas case, non constant heat capacity ratio $\left(\gamma_{\text {Air }}\right.$ and $\left.\gamma_{R 22}\right)$ as well as compressibility factors depend on temperatures that are calculated through polynomials found in [26]. At the initial state, heat capacity ratios in the region I corresponds to $\gamma_{A i r, I}=1.399$ and $\gamma_{R 22, I}=1.175$. The post-shock conditions become $T_{I I}=339.74 \mathrm{~K}, u_{I I}=115.85 \mathrm{~m} / \mathrm{s}$ and $p_{I I}=159117 \mathrm{~Pa}$ however rather close to the ideal case.

All the simulations are performed on a structured grid using, however, an adap- 


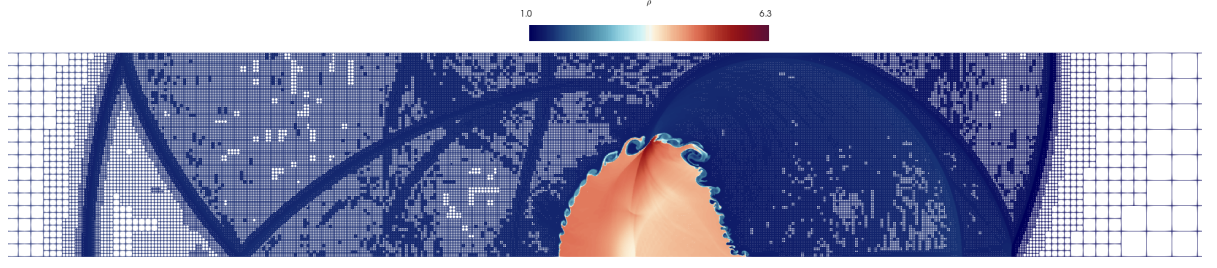

Figure 9: Solution obtained for the ideal gas configuration at a time $t=287.5 \mu$ s (dimensionless time $\left.t c_{R 22, I} / d_{0}=1.15\right)$ : Adapted grid obtained using 9 grid levels per tree (i.e. the finest mesh includes 512 grid points in each direction) and 10 trees distributed over the domain in the streamwise direction, colored by density values.

tive mesh refinement based on MRA technique rapidly described in $\S 4.3$ To get cells with an aspect ratio of unity, the computational domain is composed of 10 roots of tree data structure along the streamwise direction. Each tree comprises nine grid levels that lead to $5120 \times 512$ grid points in $(x \times y)$ directions if the solution is coded at the finest grid level. This finest grid resolution is equivalent to have 575 grid points along the initial bubble diameter $d_{0}\left(\delta x=d_{0} / 575\right)$. Denner and Wachem [30] performed a grid convergence study on the ideal gas configuration and showed that grid convergence is obtained on the finest grid they used with $\delta x=d_{0} / 500$. In the following, present simulations are performed with a CFL number of 0.5 , and a threshold MRA parameter $\sigma=10^{-3}$.

The solution of the ideal gas configuration, obtained at a time $t=287.5 \times 10^{-6}$ $\mathrm{s}$ (corresponding to a dimensionless time based on the R22 initial speed of sound $c_{R 2, I}$ of $\left.t c_{R 22, I} / d_{0}=1.15\right)$ is presented in Figure 9 where we can see the adapted grid colored by the density field. Regions where high gradients occur are clearly evidenced by the presence of a high grid level. On the opposite, in regions where a regular solution occurs, the grid is coarsened, and cells are discarded from the graded tree, leading to a drastic memory compression compared to the finest unique grid, with a reduction of $85 \%$ of the number of cells compared to the finest mesh at the final dimensionless time $t c_{R 22, I} / d_{0}=1.15$.

When the incident shock wave interacts with the R22 bubble, reflection and diffraction of the shock wave occur on the bubble interface as well as wave transmission through the bubble interface. To better describe the flow patterns observed in Figure 9, a schematic flow organization is proposed in Figure 10. When the incident shock wave hits the upstream interface (UI) of the bubble, a part of the pressure jump is transmitted inside the bubble while another part is reflected upstream. Let us mention that this reflection of the incident shock wave on the upstream bubble interface (UI) has been omitted in this schematic view but is clearly visible in front of the bubble in Figure 9 The incident shock wave passing in Air around the bubble is diffracted along the bubble surface while the refracted shock wave (RR) moves inside the bubble. This refracted shock wave interacts with the downstream bubble interface (DI), creating both a shock wave transmitted inside Air (TR) downstream the bubble and a reflected shock wave moving upstream inside the bubble. As the speed of sound of R22 is lower than in Air, the transmitted shock wave (TR) finally stays upstream of the incident shock wave (INC) (See Figure 10). Finally, the diffracted shock wave reflects on the symmetry line creating two branches of the incident shock wave (DIF), mimicking branches of the diffracted shock wave coming from the lower and 


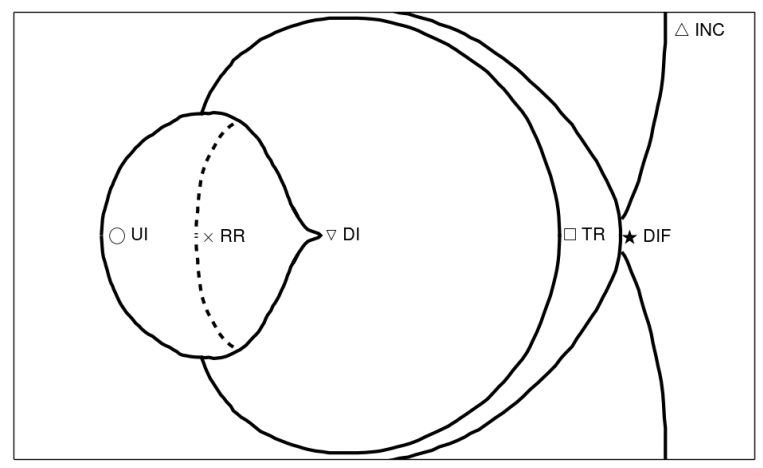

Figure 10: Schematic organization of flow patterns in shock wave/bubble interaction: UI $=$ upstream bubble interface; $D I=$ downstream bubble interface; $R R=$ regular shock wave reflection on $D I ; I N C=$ incident shock wave; $T R=$ transmitted shock wave; DIF = wave diffraction, crossing of the two branches of the incident shock

the upper sides of the bubble, crossing the symmetry plane as experimentally recorded [29].

As the high-pressure jump imposed by the incident shock wave interacts with the density jump located at the bubble interface, Richtmyer-Meshkov instabilities are distinctly visible in Figure 9 along the bubble interface. These instabilities are characteristic of the acceleration of an interface between two fluids at different densities. By comparing present results on the density field with results obtained from [30], we can see that $7^{\text {th }}$-order OSMP scheme coupled with the MRA technique produces very accurate results with a high quality of the solution mainly in the Richtmyer-Meshkov instability development.

To better assess present results, we compare them to converged results obtained by Denner and Wachem [30] on streamwise distributions of the density recorded at $y=5 \times 10^{-3} \mathrm{~m}$ (See Figure 11), and at several times $t=170 \times 10^{-6}, 222.5 \times$ $10^{-6}$, and $287.5 \times 10^{-6} \mathrm{~s}$ (corresponding to dimensionless times $t c_{R 22, I} / d_{0}=$ $0.68,0.89$, and 1.15$)$. We can see that a perfect agreement is achieved on the ideal gas configuration between our results obtained with the $7^{\text {th }}$-order OSMP scheme and those obtained by Denner and Wachem [30].

We also performed simulations of the real gas configuration to provide original results that we expect could constitute reference results. These simulations have been run with and without the proposed modified OSMP scheme. A comparison of the temperature fields obtained with and without the proposed modification is presented in Figure 12. Without the proposed modification, spurious oscillations appear on the temperature field at the upstream interface of the R22 bubble (Figure 12). One more time, we notice that the new M-P criterion on $\widetilde{\alpha}_{N+1}$ with equations (40), and (41) cure these spurious oscillations, and improve the resolution of the mass fraction discontinuities. This confirms that the M-P condition on the supplementary invariant $\widetilde{\alpha}_{N+1}$ is needed to recover a stable solution with a unique value of the compressibility factors. 

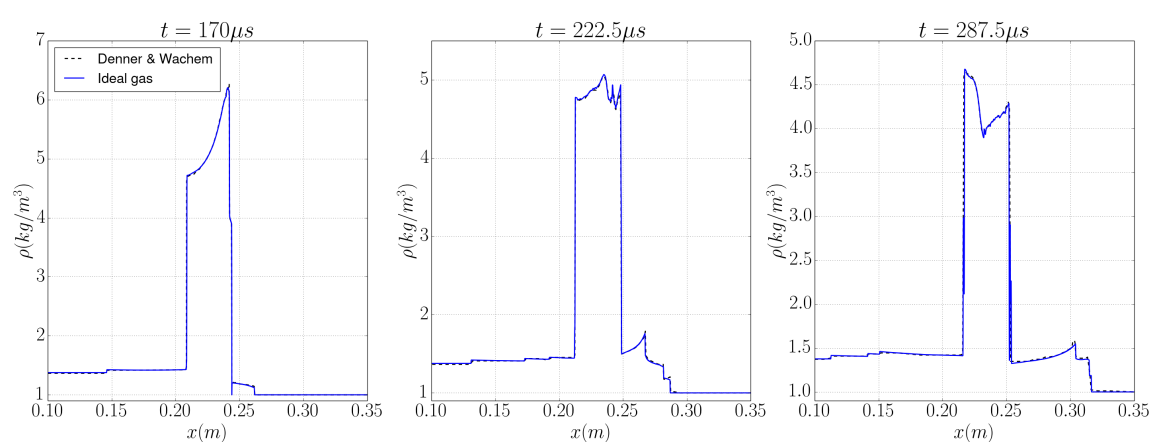

Figure 11: Streamwise distributions of the density recorded at the altitude $y=5 \times 10^{-3} \mathrm{~m}$ and at several times $t=170 \times 10^{-6}, 222.5 \times 10^{-6}$, and $287.5 \times 10^{-6} \mathrm{~s}$ : ideal gas simulation (blue bold line) is compared to results obtained by Denner and Wachem [30] (black dashed line).

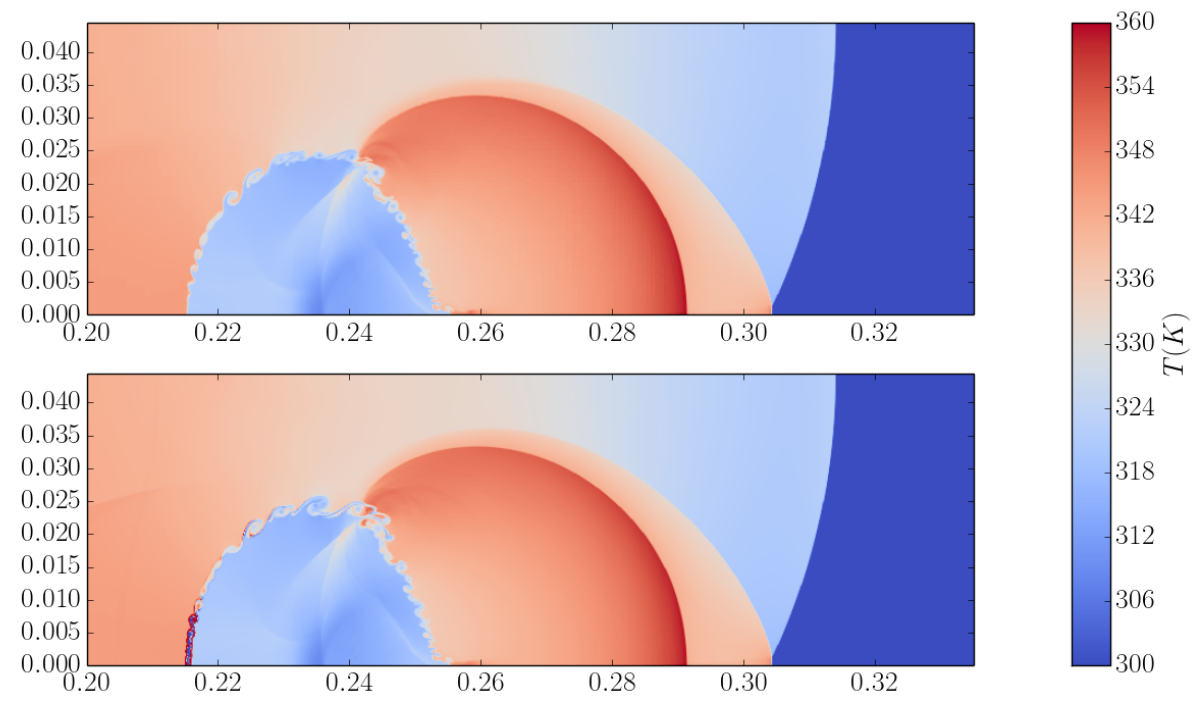

Figure 12: Temperature fields obtained with the $7^{\text {th }}$-order OSMP scheme with (at the top), and without (at the bottom) the proposed modification of the OSMP scheme. 

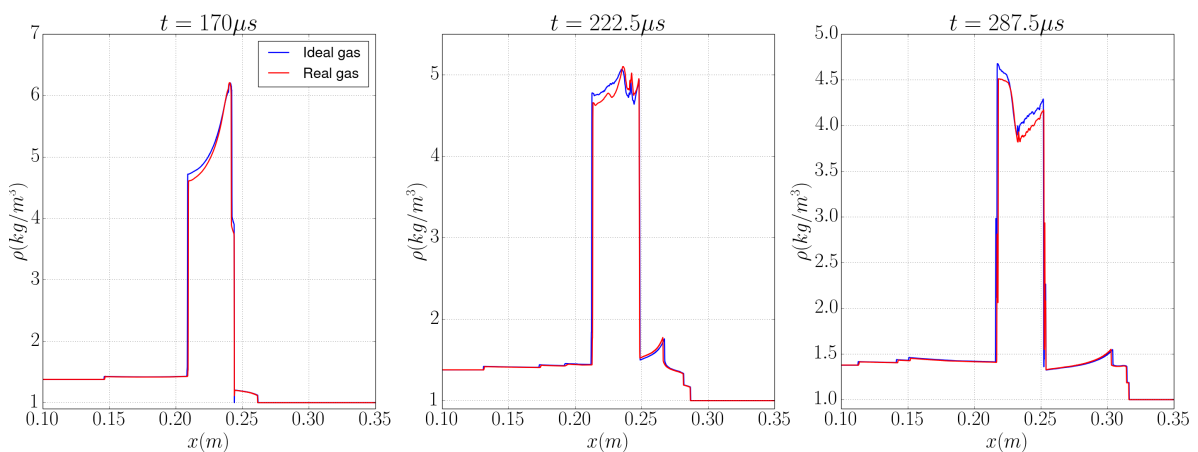

Figure 13: Streamwise distributions of the density recorded at the altitude $y=5 \times 10-{ }^{-3} \mathrm{~m}$ at several times $t=170 \times 10^{-6}, 222.5 \times 10^{-6}$, and $287.5 \times 10^{-6}$ s: ideal gas simulation (bold blue line) is compared to results for real gas configuration obtained with proposed corrected OSMP scheme (bold red line).

Although the solution for real gas flow looks like the ideal gas one, some discrepancies may be observed. Comparing real gas and ideal gas configurations at several computational times $t=170 \times 10^{-6}, 222.5 \times 10^{-6}$, and $287.5 \times 10^{-6}$, on streamwise density distributions at $y=5 \times 10^{-3} \mathrm{~m}$ (See Figure 13), we outline these differences although, as we pointed out earlier, weak differences exist between initial states. In fact, these discrepancies are concentrated in the bubble where R22 gas is present because thermodynamics coefficients of R22 real gas are strongly sensitive to the temperature (See Figure 3) leading to significant changes in the speed of sound for instance.

To better emphasize discrepancies in the field, isocontours of the temperature are plotted (See Figure 14) for both ideal (at the top) and real (at the bottom) gas obtained with the proposed modification of the OSMP scheme. As previously mentioned, differences are mainly concentrated within the R22 bubble and on its interface since the heat capacity ratio and the sound speed are significantly modified during the shock wave/bubble interaction compared to the ideal gas configuration. The modification of the sound speed also impacts the speed of the transmitted shock wave (TR) that stays upstream of the one predicted in ideal gas flow.

To validate present results for real gas flow, we compare them to experimental data coming from [29] on the $x-t$ diagram (See Figure 15). To follow all the wave motions along time, we keep the same symbols like the ones used in the schematic view of flow patterns (See Figure 10p. Although there is unavoidable variability in measurements, we can claim that a very good agreement is achieved by using the $7^{t h}$-order MP scheme with the proposed modification on $\widetilde{\alpha}_{N+1}$.

Finally, to better highlight differences between ideal and real gas configurations, we increased the traveling Mach number of the incoming shock wave to $M_{S}=2.5$ to enhance the temperature variations. Discrepancies on isocontours of the temperature are shown in Figure 16 where the real gas solution is at the top and the ideal one at the bottom. One more time, discrepancies are condensed within the bubble with a drastic change of the predicted temperature and the bubble flow pattern. At this point, the bubble has been compressed by the 


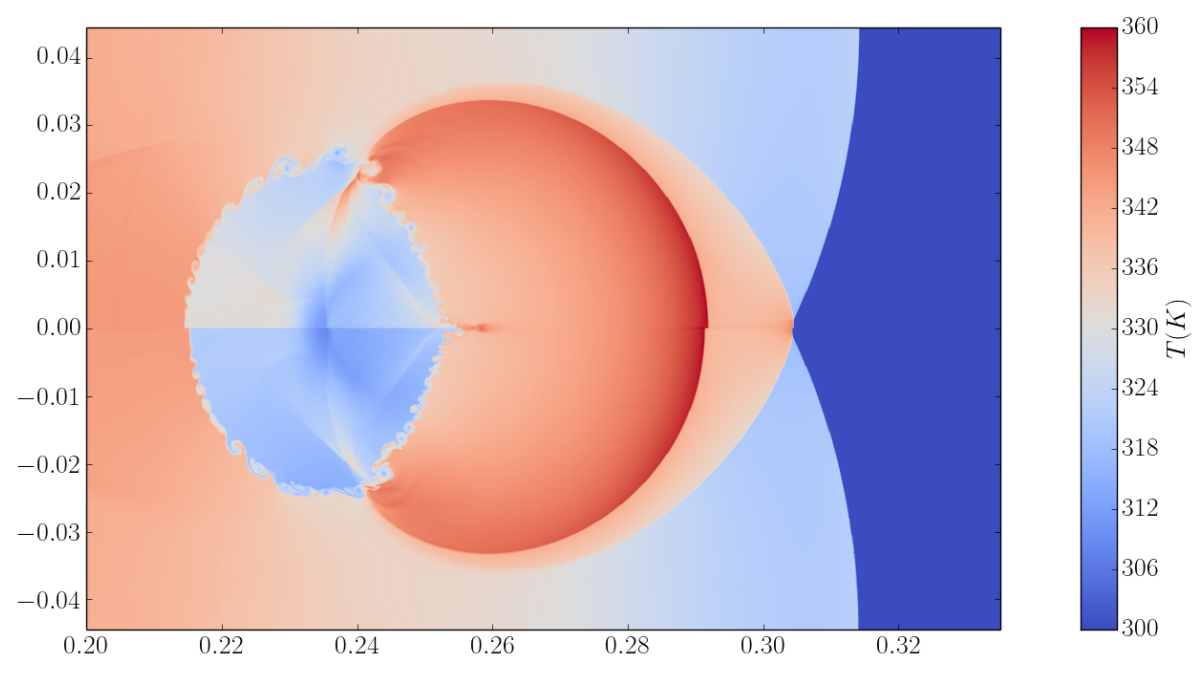

Figure 14: Isocontours of the temperature for ideal gas (at the top) and real gas (at the bottom) for $M_{s}=1.22$ at $t=287.5 \mu \mathrm{s}$.

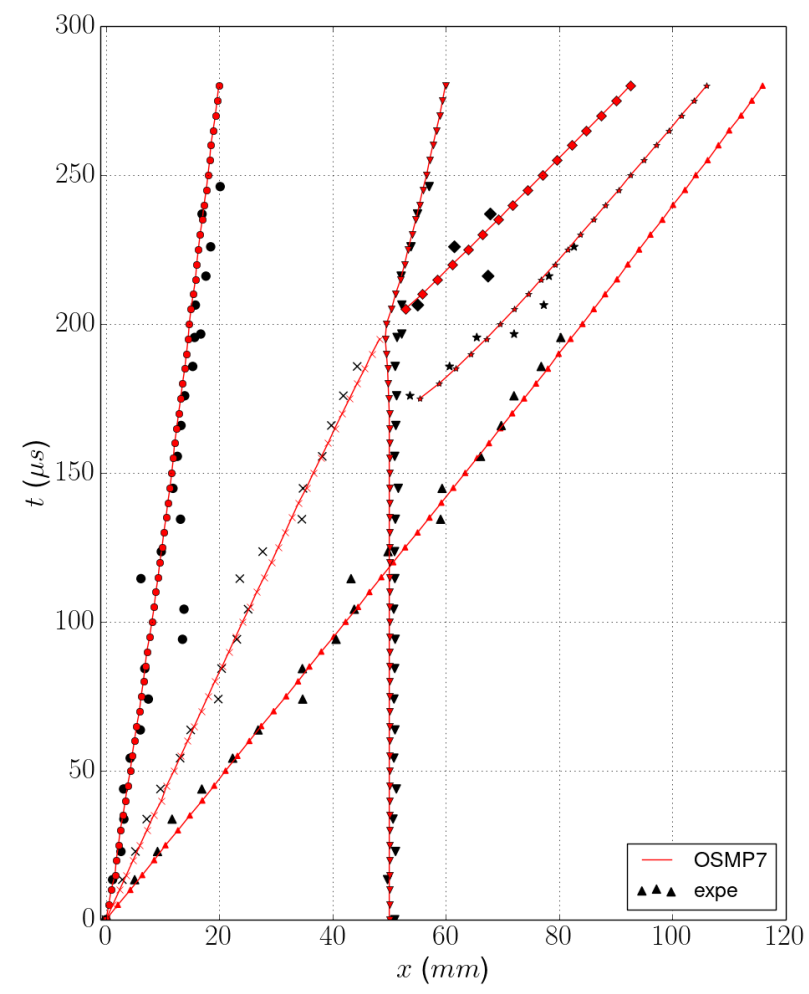

Figure 15: $x-t$ diagram of the shock wave / R22 bubble interaction. Black dots are for experiments and lines with colored dots for simulations. Symbols related to the different waves are given in Figure 10 


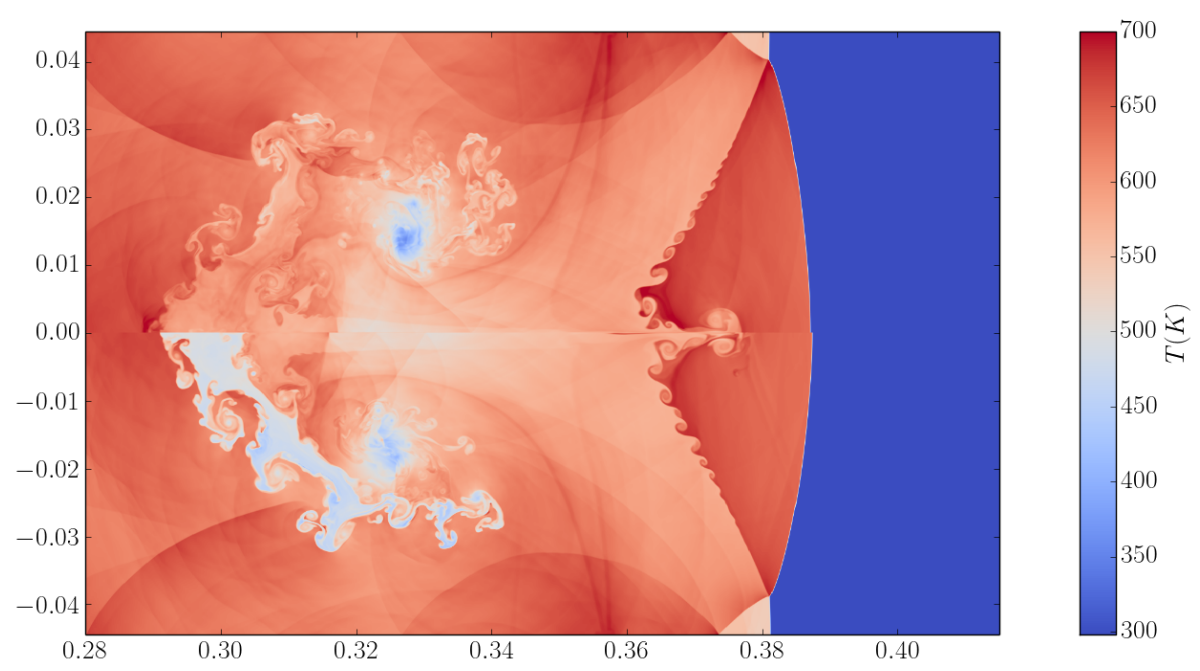

Figure 16: Isocontours of the temperature for ideal gas (at the top) and real gas (at the bottom) for $M_{s}=2.5$ at $t=250 \mu \mathrm{s}$.

shock along the x-direction while the extremities have drifted forward with the generation of vorticity accentuated by interaction with numerous acoustic waves induced by the refracted shock inside the bubble.

Figure 17 presents the evolution of the integrated value of the vorticity $(\nabla \times \vec{u})$ and the baroclinic torque term $(\nabla P \times \nabla \rho)$ on the upper half of the computational volume along time. We distinguish several phases in the evolution of these terms. At $t=0 \mathrm{~s}$, corresponding to the first interaction between the shock and the bubble, negative vorticity is created in the upper half. The baroclinic term becomes positive when the shock comes out of the bubble and reduces vorticity. We then observe a progressive decrease of the vorticity generation disturbed by multiple refracted shock waves in the computational volume, which still interacts with the bubble. We observe those vorticity productions in Figure 18 with the evolution of the isocontours of R22 mass fraction at different times. Multiple vortex sizes resulting from successive interactions with shock and acoustic waves are visible. It should be noted that because of the absence of viscosity in the $2 \mathrm{D}$ simulation, the size of the smaller vortex is only limited by the size of the mesh.

All those different aspects are visible in Figure 19 representing the density isolines after $250 \mu \mathrm{s}$ of simulation. We can observe the main shock waves, the perturbations induced by the multiple reflected shock waves through the bubble and the vortex, and the region of mixing between the high-density R22 gas and the air.

\section{Conclusions}

In this paper, we developed a stable high-order Roe scheme capable of predicting multicomponent real gas flows devoted to applications in hypersonic aerodynamics or detonation configurations where shock waves with high-temperature jumps 

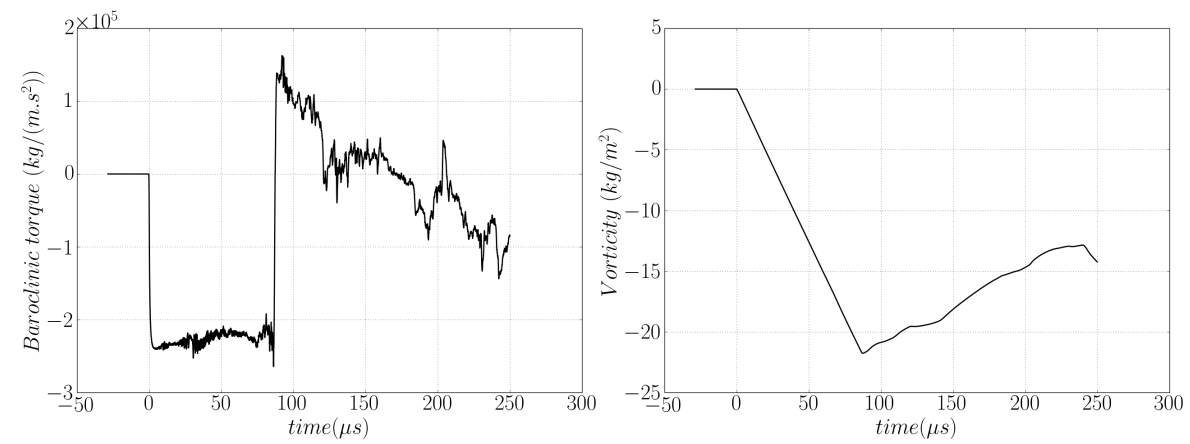

Figure 17: Baroclinic torque (left) and vorticity (right) as a function of time integrated on the computational volume corresponding to the upper half of the total volume.
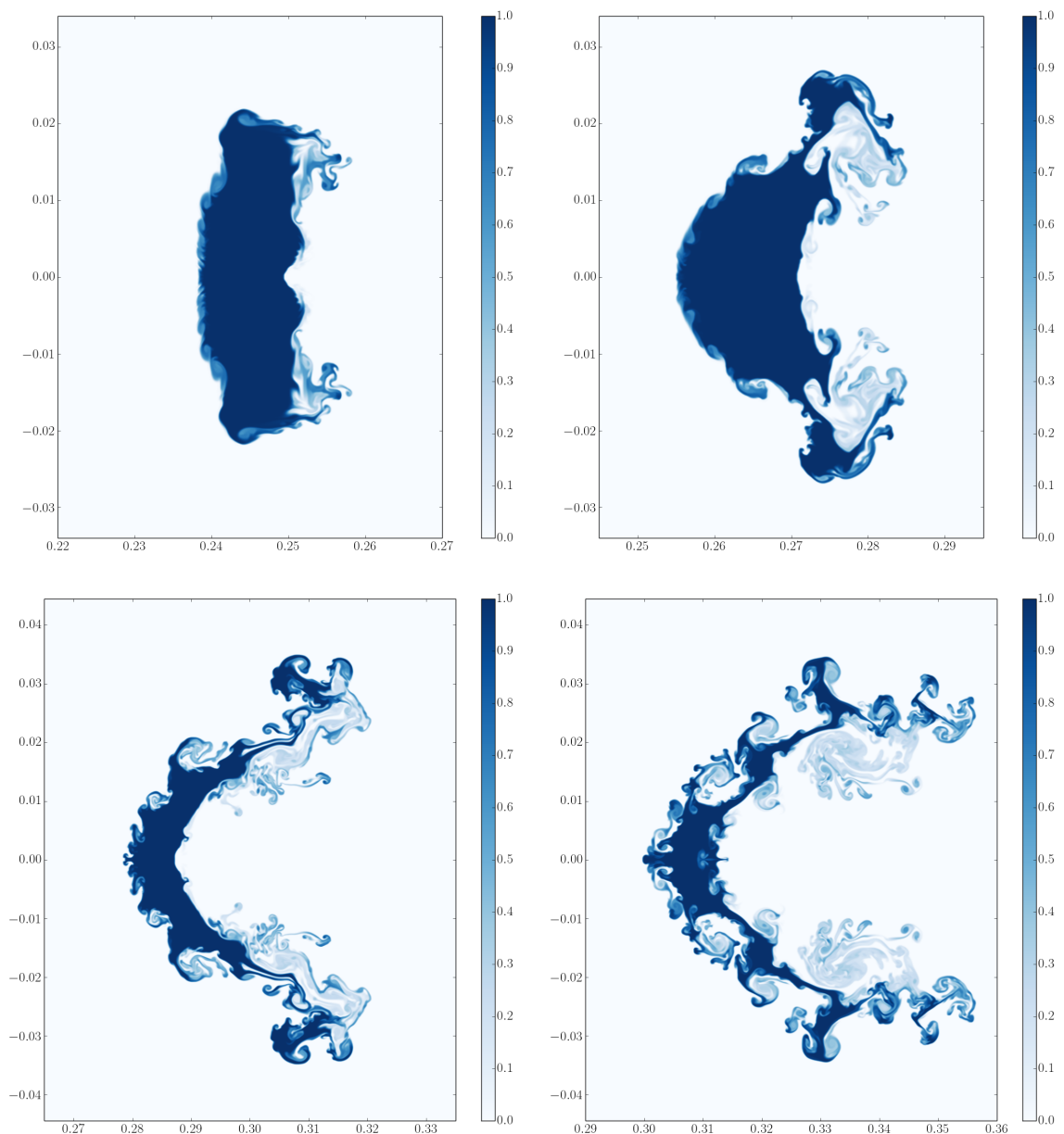

Figure 18: Isocontours of mass fraction of R22 gas for a $M_{s}=2.5$ shock wave at $t=100 \mu \mathrm{s}$, $t=150 \mu \mathrm{s}, t=200 \mu \mathrm{s}$ and $t=250 \mu \mathrm{s}$. Symmetry is applied from the computation on the upper half to obtain image of the full bubble. 


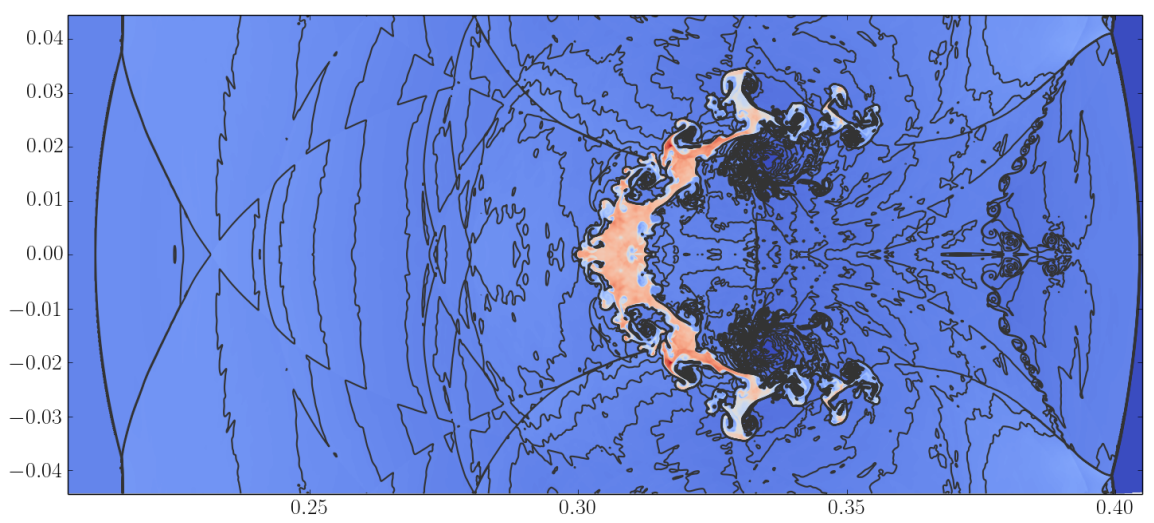

Figure 19: Isocontours and Isolines of density from 1.2 to $10 \mathrm{~kg} / \mathrm{m}^{3}$ with level every 0.2 $\mathrm{kg} / \mathrm{m}^{3}$ for a $M_{s}=2.5$ shock wave at $t=250 \mu \mathrm{s}$.

interact with mixtures of several species. We first proposed a generalization of the Roe solver for distinct species with multiple thermodynamic properties for a mixture of non-ideal gases that relies on the original method proposed by Vinokur \& Montagné [9]. It first consists in obtaining a predicted Roe averaged value of the compressibility factors using the left and right thermodynamic states of the Riemann problem, then corrects these predictions by orthogonal projection onto the hyperplane defined by the relationship between the pressure jump and the compressibility factors of all species. Our proposed method allows us to approximate an averaged speed of sound that is coherent with the Roe average of the Riemann problem. We secondly introduce the proposed Roe averaged state of the compressibility factors into the One-Step Monotonicity-Preserving (OSMP) scheme to obtain an extension of the high-order Lax-Wendroff procedure towards real gas flows. However, the Roe averaged compressibility factors calculated at cell interfaces can be inconsistent with the mass fraction along the wide stencil required by the high-order approximation. This can lead to a low order of convergence and to numerical artifacts that appear when large variations of species occur, for instance, in the vicinity of contact discontinuities. To overcome those drawbacks, we proposed a new formulation of the total energy flux based on a combination of the Riemann invariants relative to the multispecies. This new formulation has the advantage of not using the independent values of the compressibility factors and avoiding error dependency with approximation created in the extension of Vinokur and Montagné method for multispecies. Knowing the averaged speed of sound estimated by our proposed extension of the Vinokur \& Montagné method, we demonstrate that the proposed new formulation is equivalent to defining a constraint on the compressibility factors that completely fulfill the jump relationships of the Riemann problem. We also showed on well-documented 1-D and 2-D test cases that this proposed modification avoids numerical artifacts and enforces uniqueness of the averaged value of the compressibility factors.

To properly resolve discontinuities while optimizing the number of numerical cells, the high-order Monotonicity-Preserving numerical scheme equipped with 
the proposed constraint on compressibility factors is combined with an Adaptive Multiresolution procedure to automatically refine grid points in regions where steep gradients occur and coarsen grid points elsewhere. The present numerical method is validated with mixtures of non-calorically perfect ideal gases with temperature-dependent heat capacities. OSMP scheme applies on the new combination of Riemann invariants allows preserving high-order of accuracy in smooth solutions despite large variations of composition and temperature. In classical shock tube problem applied to non-ideal gas, it efficiently captures the mass fraction contact discontinuities with robustness and avoids numerical artifacts. Non-ideal gas effects are then pointed out mainly on the modification of the speed of sound with the temperature that induces discrepancies on the wave speeds as well as on the constant states between waves, compared to ideal gas configuration. A 2-D interaction between a shock wave in Air with a cylindrical bubble initially filled with dense refrigerant R22 gas is also considered. Present results accurately recover the fully resolved recent numerical solution of calorically perfect gases. They also compare well with experimental results obtained with (of course) real gases. We finally performed simulations of a real gas configuration with a higher Mach number leading to higher temperature variations. Results clearly exhibit discrepancies between ideal and real gas configurations condensed within the bubble since the R22 refrigerant gas has thermodynamics properties that are mainly sensitive to temperature variations. Thus, drastic changes are recorded on the predicted temperature and the bubble flow patterns that fully justify the use of relevant thermodynamics and the proposed numerical method to account for real gas properties.

This study constitutes a first step towards the development of numerical tools able to predict shocked reacting flows encountered, for instance, in DeflagrationDetonation Transition problems. Therefore, in the near future, the proposed numerical procedure will be integrated into a Navier-Stokes code capable of predicting with both robustness and high accuracy multicomponent reactive flows with compressible effects.

\section{Acknowlegments}

This work is supported by the French Alternative Energies and Atomic Energy Commission. Computational facilities were provided by the Institute for Development and Resources in Intensive Scientific Computing.

\section{References}

[1] J.-L. Montagne, H. C. Yee, M. Vinokur, Comparative study of highresolution shock-capturing schemes for a real gas, AIAA Journal 27 (10) (1989) 1332-1346.

[2] V. Daru, C. Tenaud, High order one-step monotonicity-preserving schemes for unsteady compressible flow calculations, Journal of Computational Physics 193 (2004) 563-594.

[3] A. Cohen, S. M. Kaber, S. Müller, M. Postel, Fully adaptive multiresolution finite volume schemes for conservation laws, Math. Comp. 72 (2003) 183225 . 
[4] J. Anderson, J. D., Hypersonics and High Temperature Gas Dynamics, McGraw-Hill Book Company, New-York, 1989.

[5] T. Poinsot, D. Veynante, Theoretical and numerical combustion, Prog. Energy Combust. Sci. 28 (01 2005).

[6] P. Roe, Approximate riemann solvers, parameter vectors, and difference schemes, Journal of Computational Physics 43 (2) (1981) 357 - 372.

[7] R. Abgrall, An extension of roe's upwind scheme to algebraic equilibrium real gas models, Computers \& Fluids 19 (2) (1991) $171-182$.

[8] P. Glaister, An approximate linearised riemann solver for the euler equations for real gases, Journal of Computational Physics 74 (2) (1988) 382 408.

[9] M. Vinokur, J.-L. Montagné, Generalized flux-vector splitting and roe average for an equilibrium real gas, Journal of Computational Physics 89 (2) (1990) $276-300$.

[10] M.-S. Liou, B. V. Leer, J.-S. Shuen, Splitting of inviscid fluxes for real gases, Journal of Computational Physics 87 (1) (1990) 1-24.

[11] C. F. Cox, P. Cinnella, General solution procedure for flows in local chemical equilibrium, AIAA Journal 32 (3) (1994) 519-527.

[12] S. Arabi, J.-Y. Trépanier, R. Camarero, A simple extension of roe's scheme for real gases, Journal of Computational Physics 329 (2017) 16 - 28.

[13] S. P. Spekreijse, R. Hagmeijer, Derivation of a roe scheme for an n-species chemically reacting gas in thermal equilibrium, in: P. Wesseling (Ed.), Proceedings of the Eighth GAMM-Conference on Numerical Methods in Fluid Mechanics, Vieweg+Teubner Verlag, Wiesbaden, 1990, pp. 522-532.

[14] L. Mottura, L. Vigevano, M. Zaccanti, An evaluation of roe's scheme generalizations for equilibrium real gas flows, Journal of Computational Physics 138 (2) (1997) $354-399$.

[15] S. Arabi, J.-Y. Trépanier, R. Camarero, A simple extension of roe's scheme for multi-component real gas flows, Journal of Computational Physics 388 (2019) $178-194$.

[16] B. Larrouturou, How to preserve the mass fractions positivity when computing compressible multi-component flows, Journal of Computational Physics 95 (1) (1991) $59-84$.

[17] R. Abgrall, How to prevent pressure oscillations in multicomponent flow calculations: A quasi conservative approach, Journal of Computational Physics 125 (1) (1996) $150-160$.

[18] V. Daru, C. Tenaud, Numerical simulation of the viscous shock tube problem by using a high resolution monotonicity-preserving scheme, Computers \& Fluids 38 (2009) 664-676. 
[19] A. Suresh, H. Huynh, Accurate monotonicity-preserving schemes with runge-kutta time stepping, Journal of Computational Physics 136 (1997) 83-99.

[20] G. Strang, Accurate partial difference methods. i. linear cauchy problems, Arch. Ration. Mech. Anal. 12 (1963) 392-402.

[21] R. J. Leveque, Numerical Methods for Conservation Laws, 2nd Edition, Springer, 1992.

[22] I. Ben Hassan Saïdi, G. Fournier, C. Tenaud, On the behavior of high order one-step monotonicity-preserving scheme for direct numerical simulation of shocked turbulent flows., International Journal of Computational Fluid Dynamics 34 (9) (2020) 671-704.

[23] A. Harten, Multiresolution algorithms for the numerical solution of hyperbolic conservation laws, Communications on Pure and Applied Mathematics 48 (12) (1995) 1305-1342.

[24] A. Cohen, Wavelet methods in numerical analysis, Vol. 7 of Handbook of Numerical Analysis, P.G. Ciarlet and J.L. Lions, editors, Elsevier, Amsterdam, 2000.

[25] C. Tenaud, O. Roussel, L. Bentaleb, Unsteady compressible flow computations using an adaptive multiresolution technique coupled with a high-order one-step shock-capturing scheme, Computers \& Fluids 120 (2015) 111 125.

[26] B. McBride, M. Zehe, S. Gordon, Nasa glenn coefficients for calculating thermodynamic properties of individual species, NASA (10 2002).

[27] A. Beccantini, Upwind splitting schemes for ideal gases mixtures with temperature-dependant specific heat capacities, Ph.D. thesis, Université d'Evry, Evry-Val d'Essonne (2000).

[28] F. Coquel, C. Marmignon, P. Rai, F. Renac, An entropy stable high-order discontinuous galerkin spectral element method for the baer-nunziato twophase flow model, Journal of Computational Physics 431 (2021) 110135.

[29] J.-F. Haas, B. Sturtevant, Interaction of weak shock waves with cylindrical and spherical gas inhomogeneities, Journal of Fluid Mechanics 181 (1987) 41-76.

[30] F. Denner, B. G. M. van Wachem, Numerical modelling of shock-bubble interactions using a pressure-based algorithm without riemann solvers, Experimental and Computational Multiphase Flow 1 (4) (2019) 271-285.

[31] A. Kundu, S. De, High resolution numerical simulation of a shockaccelerated refrigerant-22 bubble, Computers \& Fluids 193 (2019) 104289.

[32] T. J. Poinsot, S. K. Lele, Boundary conditions for direct simulations of compressible viscous flows, Journal of Computational Physics 101 (1992) 104-129. 